\title{
Differential Diagnostic Methods for Cognitive Disorders Using Neural Networks Based on Electroencephalogram Signals: A Systematic Review
}

Saman Fouladi, Ali asghar Safaei*

Department of Medical Informatics, Tehran School of Medical Sciences, Tarbiat Modares University, Tehran, Iran

\section{ABSTRACT}

Introduction: Alzheimer's disease is a brain disorder that gradually destroys cognitive function and eventually the ability to carry out daily routine tasks. Early diagnosis of this disease has attracted the attention of many physicians and scholars, and several methods have been used to detect it in early phases. Evaluation of artificial neural networks is low-cost with no side effect method that is used for diagnosing and predicting Alzheimer's disease in subjects with mild cognitive impairment based on electroencephalogram signals. Materials and Methods: for this systematic review, keywords Alzheimer's, Artificial Neural network and EEG were searched in IEEE, PubMed central, ScienceDirect, and Google Scholar databases between 2000 to 2019. Then, they were selected for critical evaluation based on the most relevance to the subject under study. Results: The search result in these databases was 100 articles. Excluding unrelated articles, only 30 articles were studied. In the present study, different types of artificial neural networks were described, Next, the accuracy of the classification obtained by these methods was investigated. The results have shown that some methods, despite being less used in research or have simple architecture, have the highest accuracy for classification. In many studies, artificial neural networks have also been considered in comparison with other classification methods and the results show the superiority of these methods. Conclusion: Artificial neural networks can be used as a tool for early detection of Alzheimer's disease. This approach can be evaluated for its classification accuracy, speed, architecture, and common use. Some networks are accurate at classifying and understanding data, but are slow or require specific hardware/software environments. Some other networks work better with simple architectures than complex networks. Furthermore, changing the architecture of conventional networks or combining them with other methods resulted in significantly different results. Increasing accuracy of classification of these networks in the diagnosis of mild cognitive impairment could help to predict Alzheimer's disease appropriately.

\section{Keywords:}

1. Alzheimer Disease

2. Cognitive Dysfunction

3. Electroencephalography

*Corresponding Author: Ali asghar Safaei

E-mail:aa.safaei@modares.ac.ir 


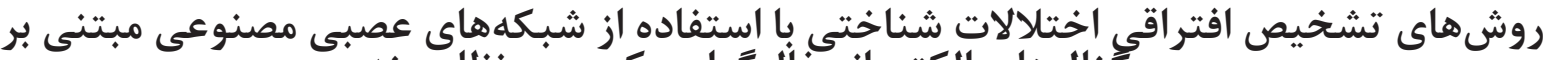

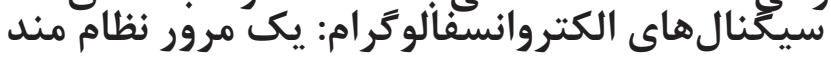

\author{
سمن فولادى، على اصغر صفايى" \\ كَروه انفورماتيك يزشكى، دانشكده علوم يزشكى تهران، دانشكاه تربيت مدرس، تهران، ايران
}

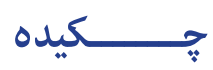

مقدمه: بيمارى آلزايمر نوعى اختلال مغز است كه به تدريج عملكرد شناختى و درو در نهايت توانايى انجام

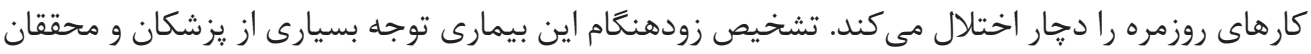

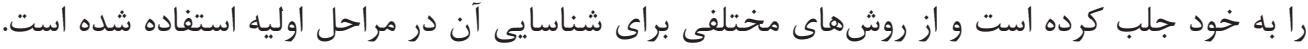

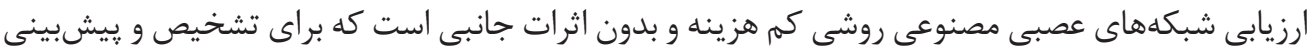

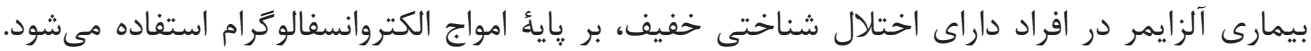

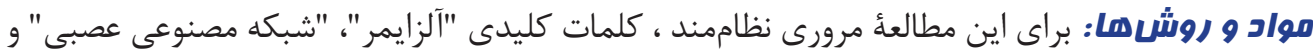

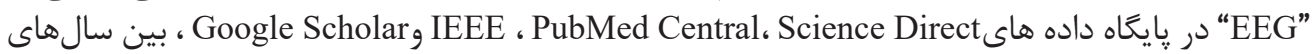

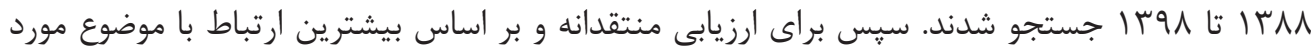

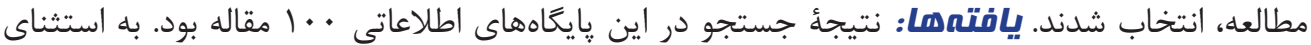

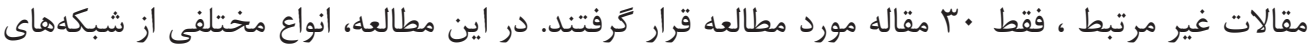

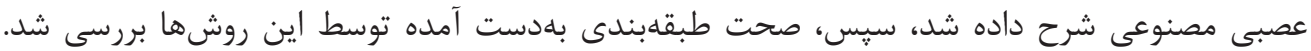

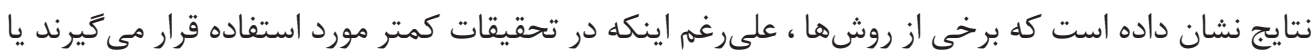

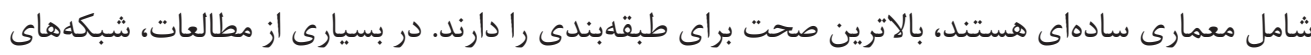

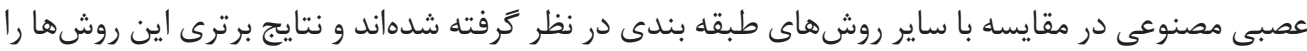

وازمهاى كليدى:

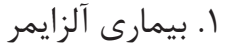

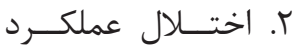

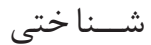

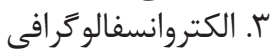

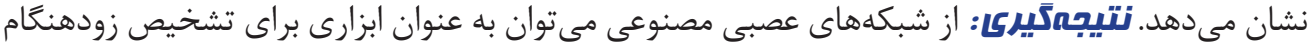

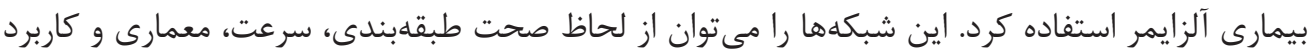

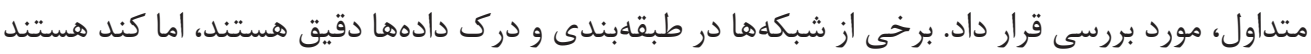

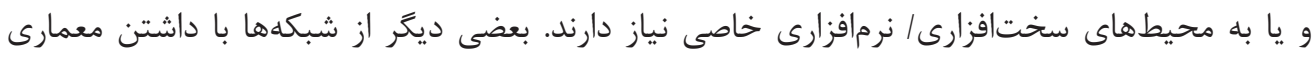

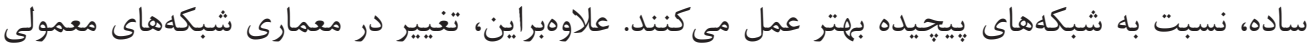

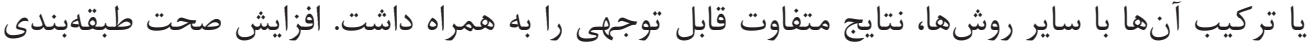

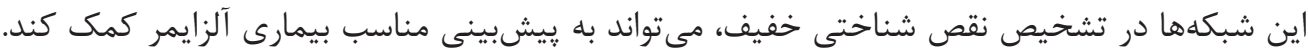




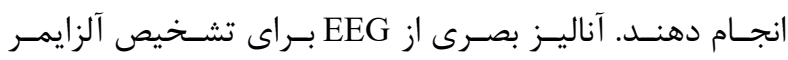

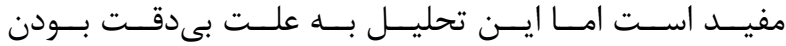

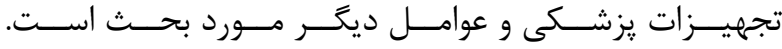

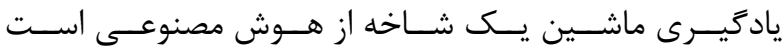

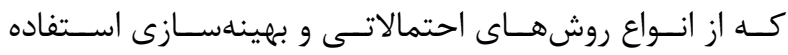

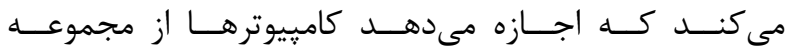

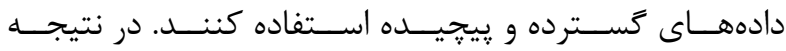

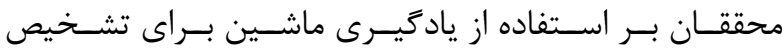

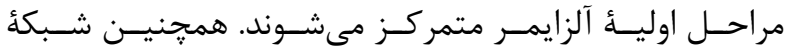

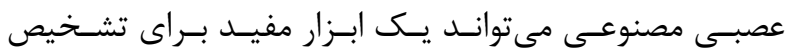

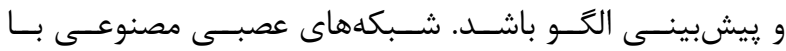

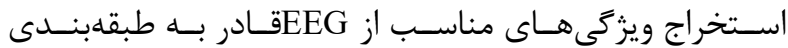

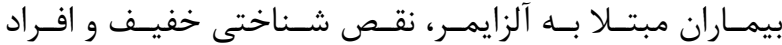

$$
\begin{aligned}
& \text { عــادى مىباشـــند. } \\
& \text { مواد و روشها }
\end{aligned}
$$

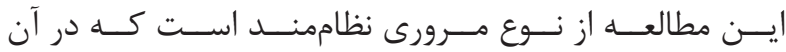

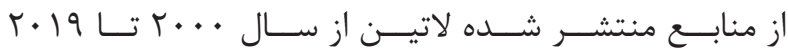

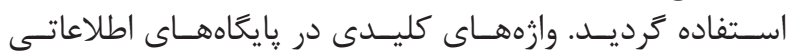
Google Scholar و Sciencedirect ،PubMed central ،IEEE

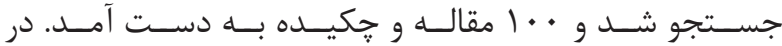

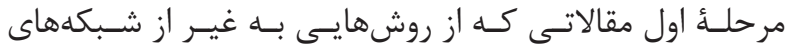

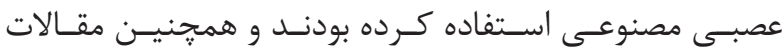

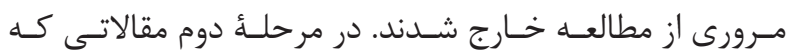

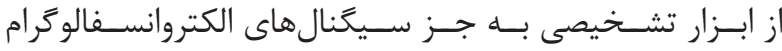

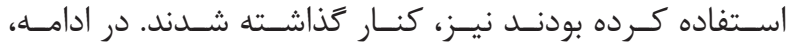

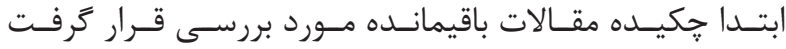

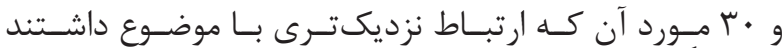

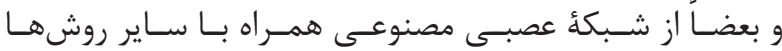

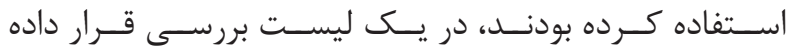

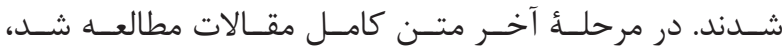

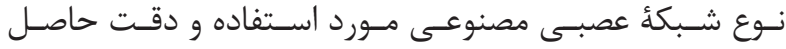

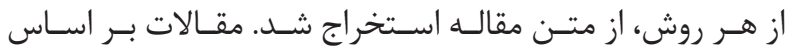

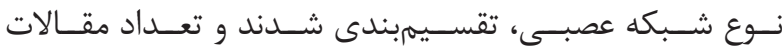

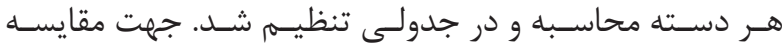

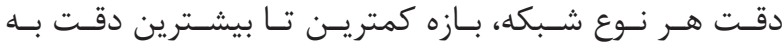

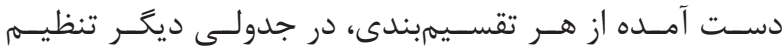

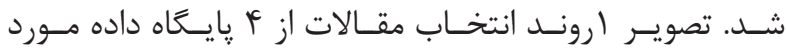

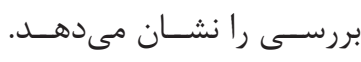

يافته ها

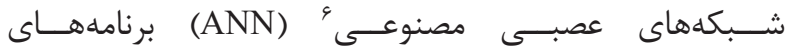

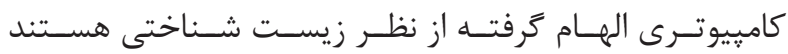

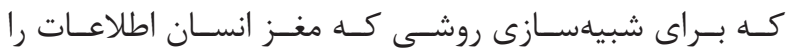

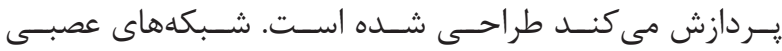

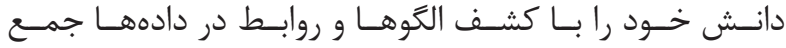

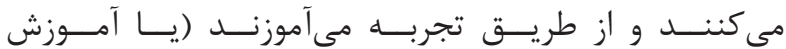

${ }^{1}$ Alzheimer's Disease; AD

${ }^{2}$ Computed Tomography

${ }^{3}$ Magnetic Resonance Imaging

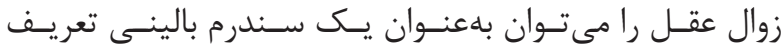

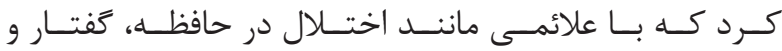

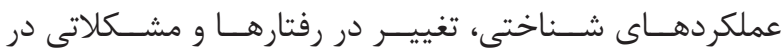

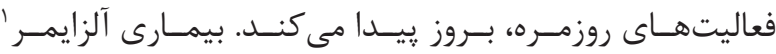

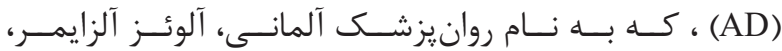

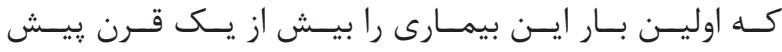

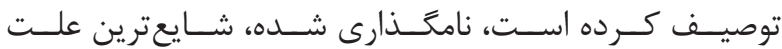

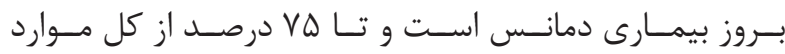

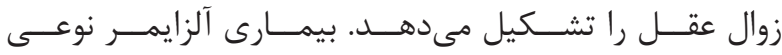

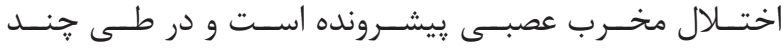

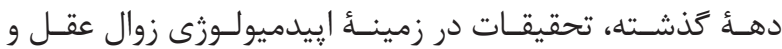

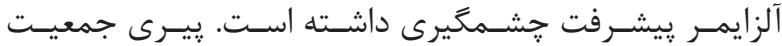

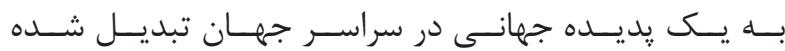

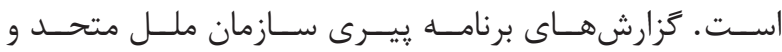

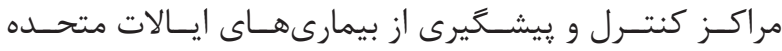

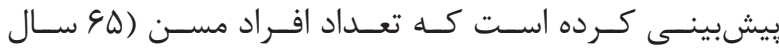

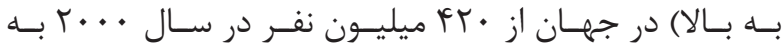

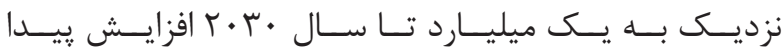

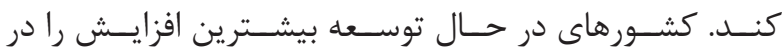

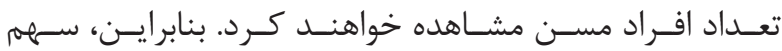

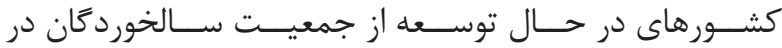

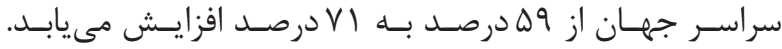

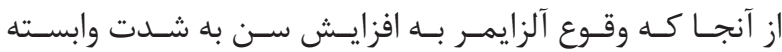

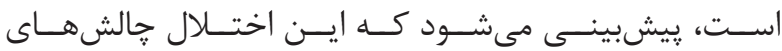

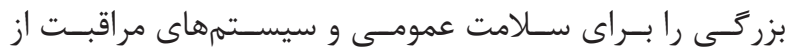

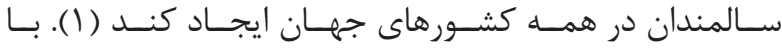

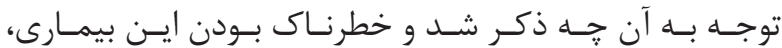

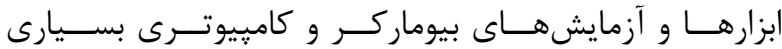

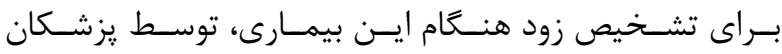

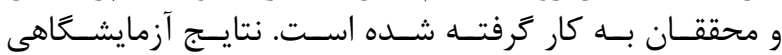

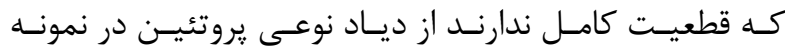

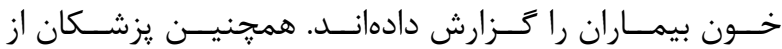

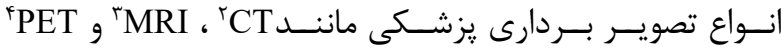

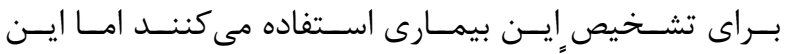

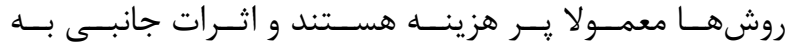

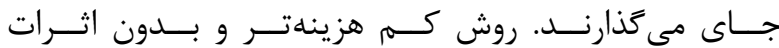

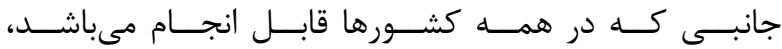

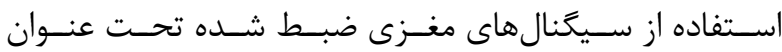

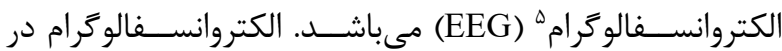

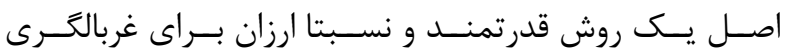

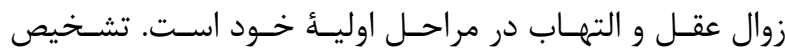

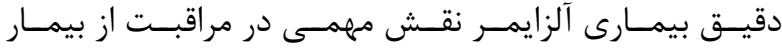

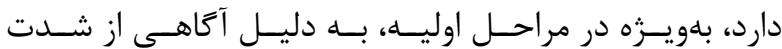

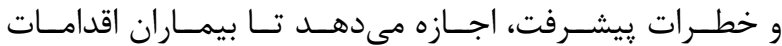

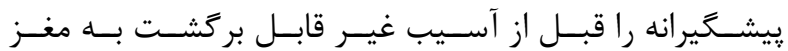

${ }^{4}$ Positron Emission Tomography

${ }^{5}$ Electroencephalogram; EEG

${ }^{6}$ Artificial neural networks; ANN 


\section{بايعاه 1Y: PubMed}

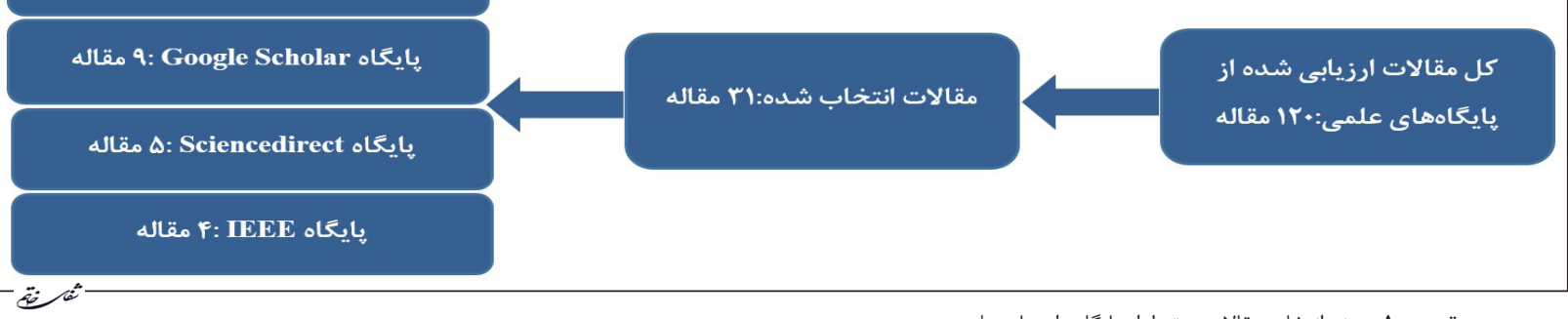

تصوير ا- روند انتخاب مقالات مرتبط از پاياًاه دادهاى علمى

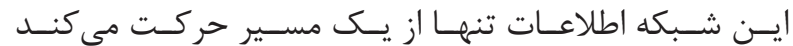

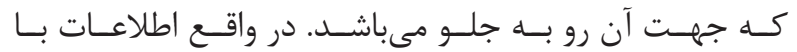

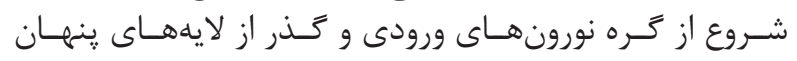

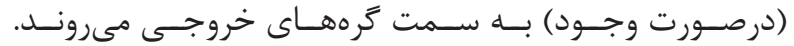

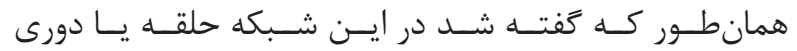

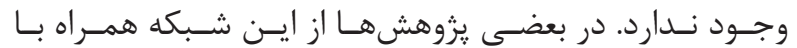

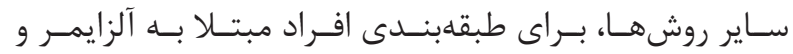

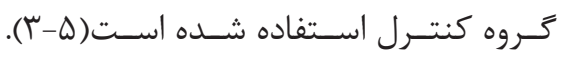

\section{شبكة عصبى :رسيرون تك لايه}

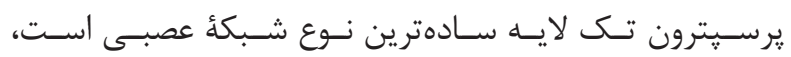

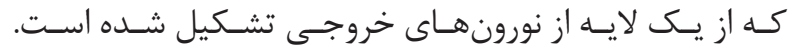

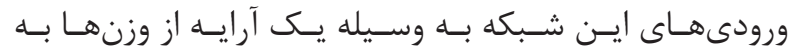

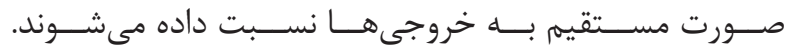

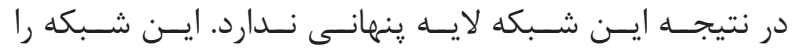

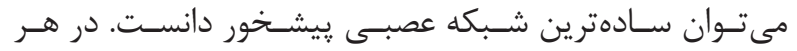

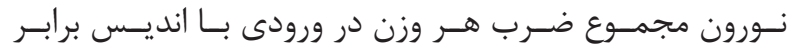

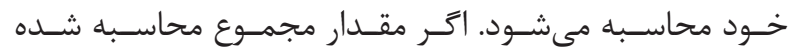

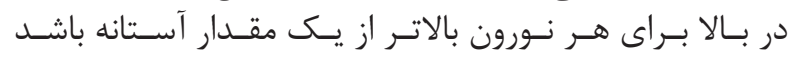

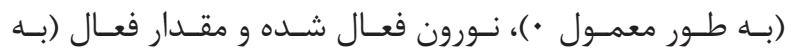

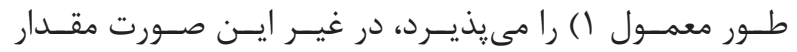

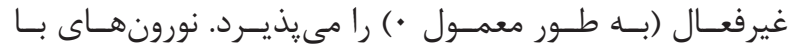

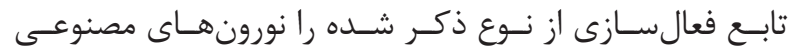

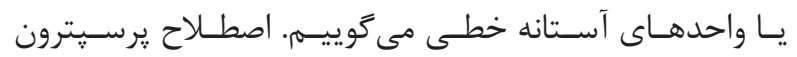

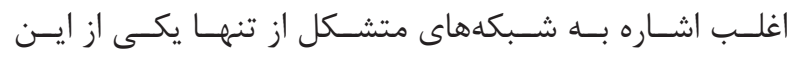

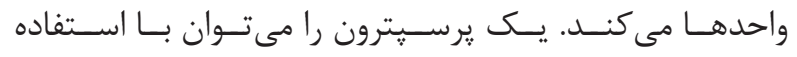

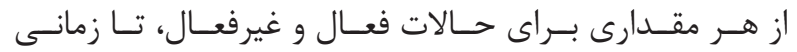

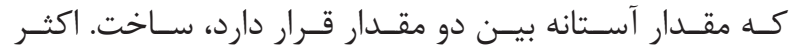

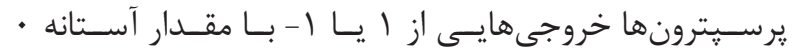

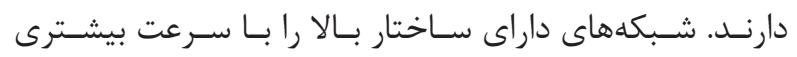

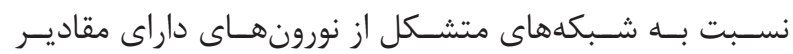

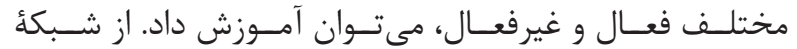

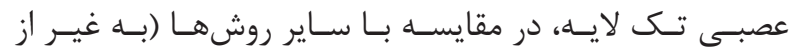

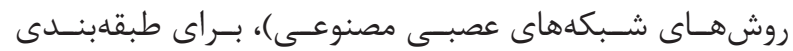

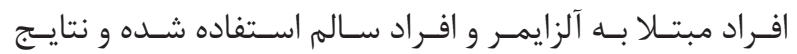

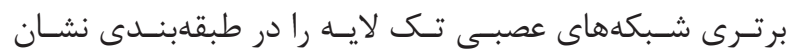

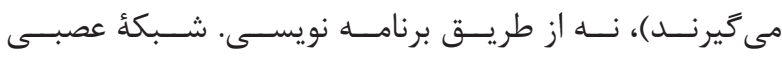

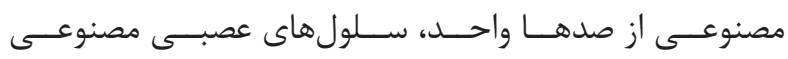

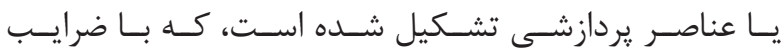

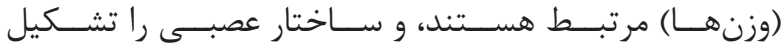

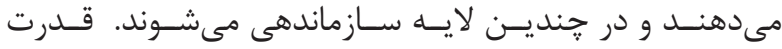

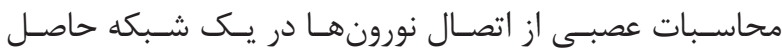

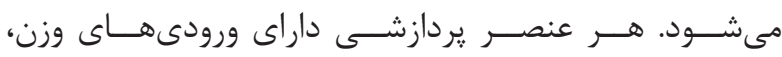

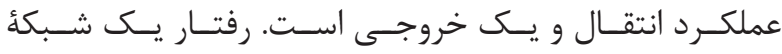

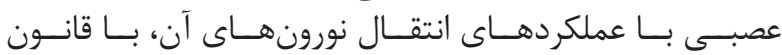

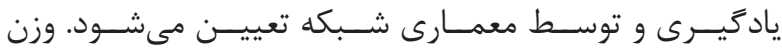

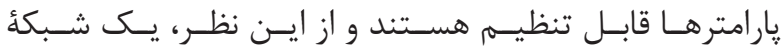

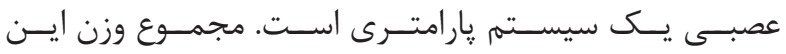

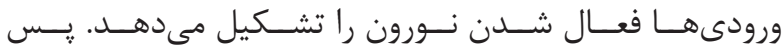

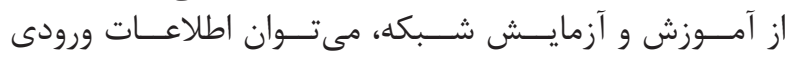

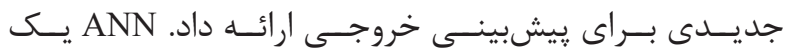

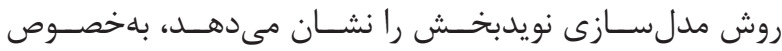

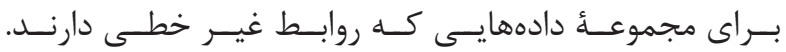

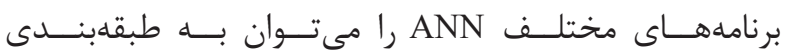

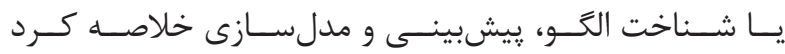

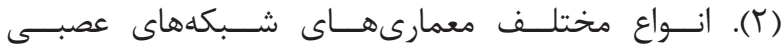

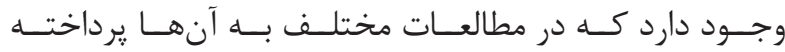

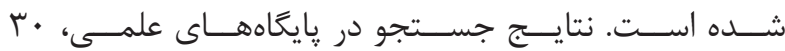

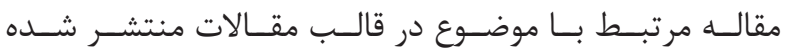

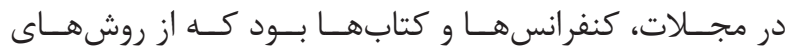

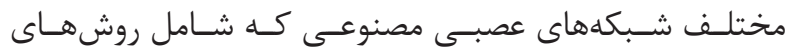

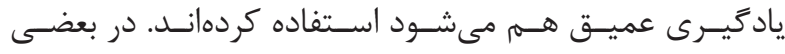

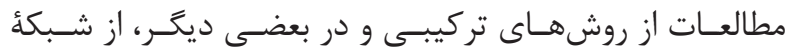

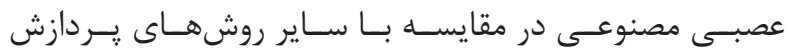

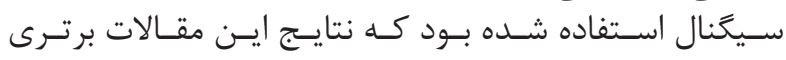

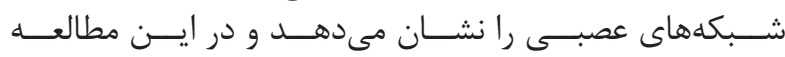

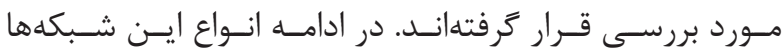

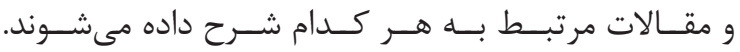

شبكة عصبى :ييشخور (FFNN)

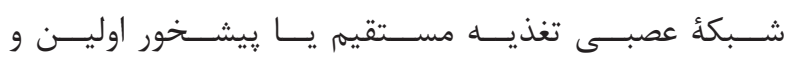

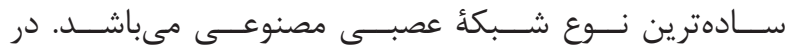




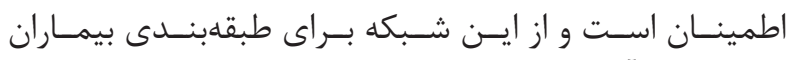

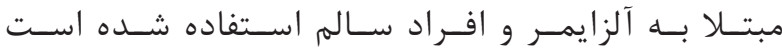

شبكؤ عصبى پِ انتشار خطا

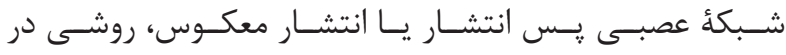

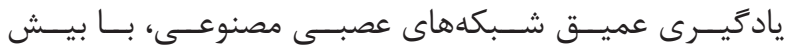

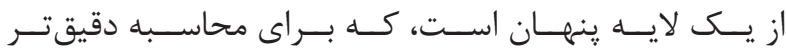

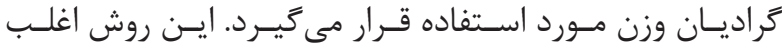

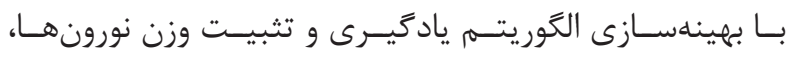

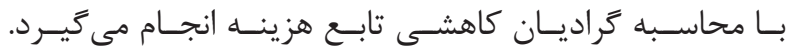

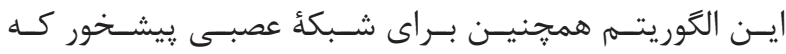

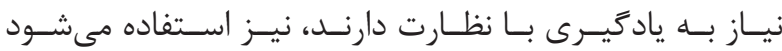

$(10-19)$

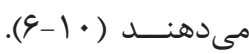

شبكة عصبى ثيرسيترون گند لايه(MLP ANN)

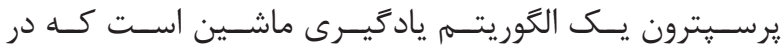

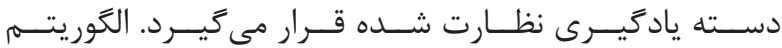

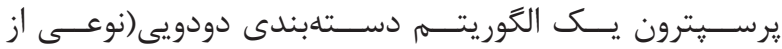

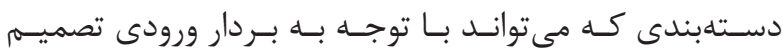

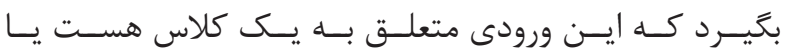

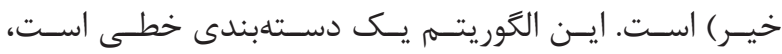

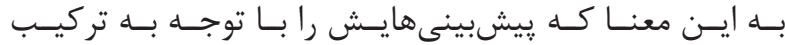

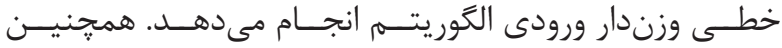

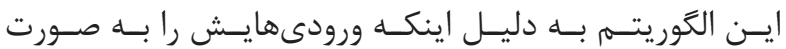

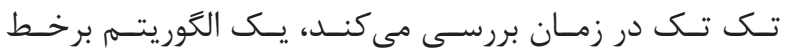

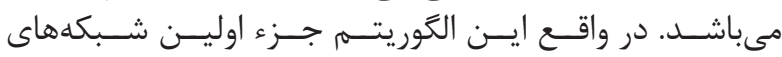

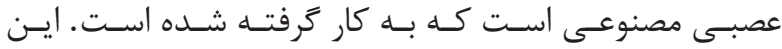

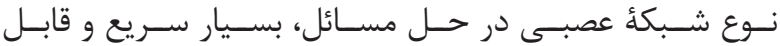

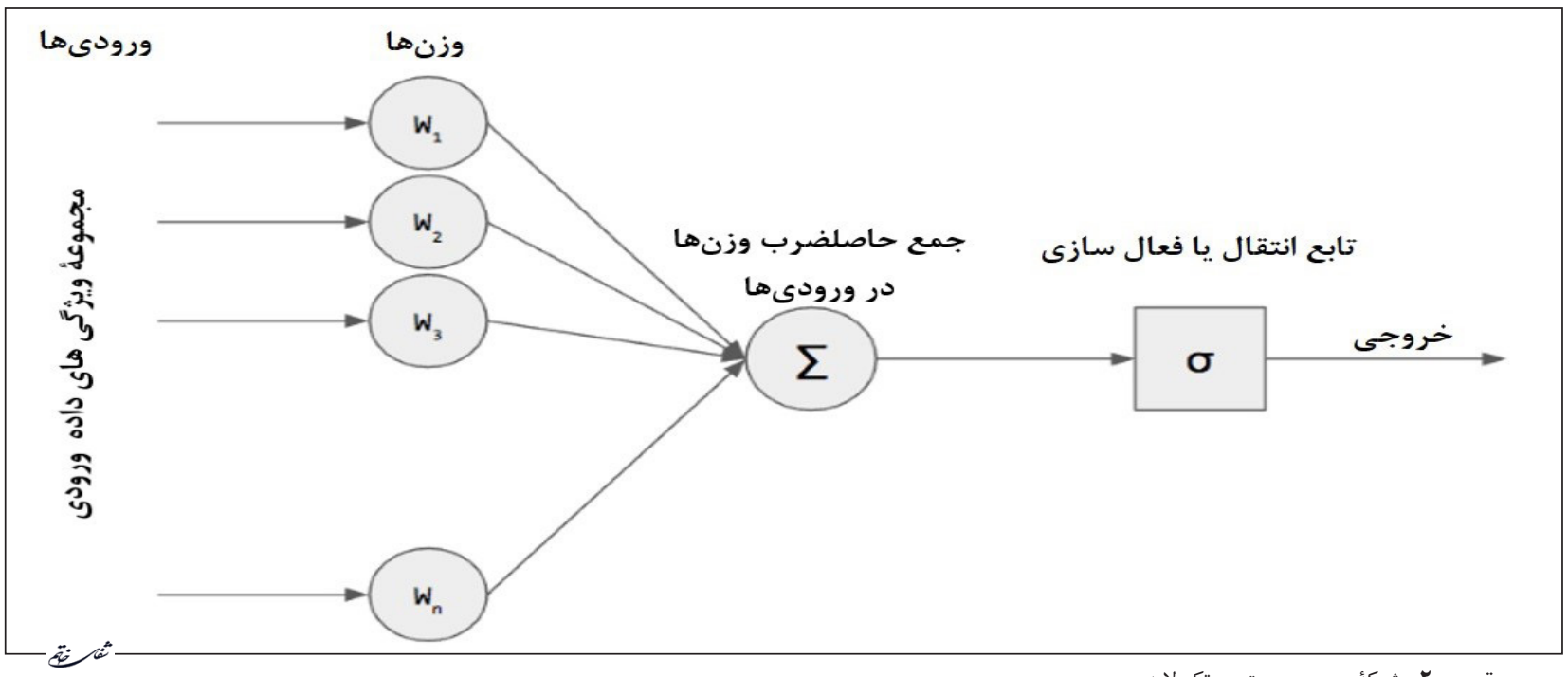

تصوير r- شبكة عصبى برسيترون تك لايه

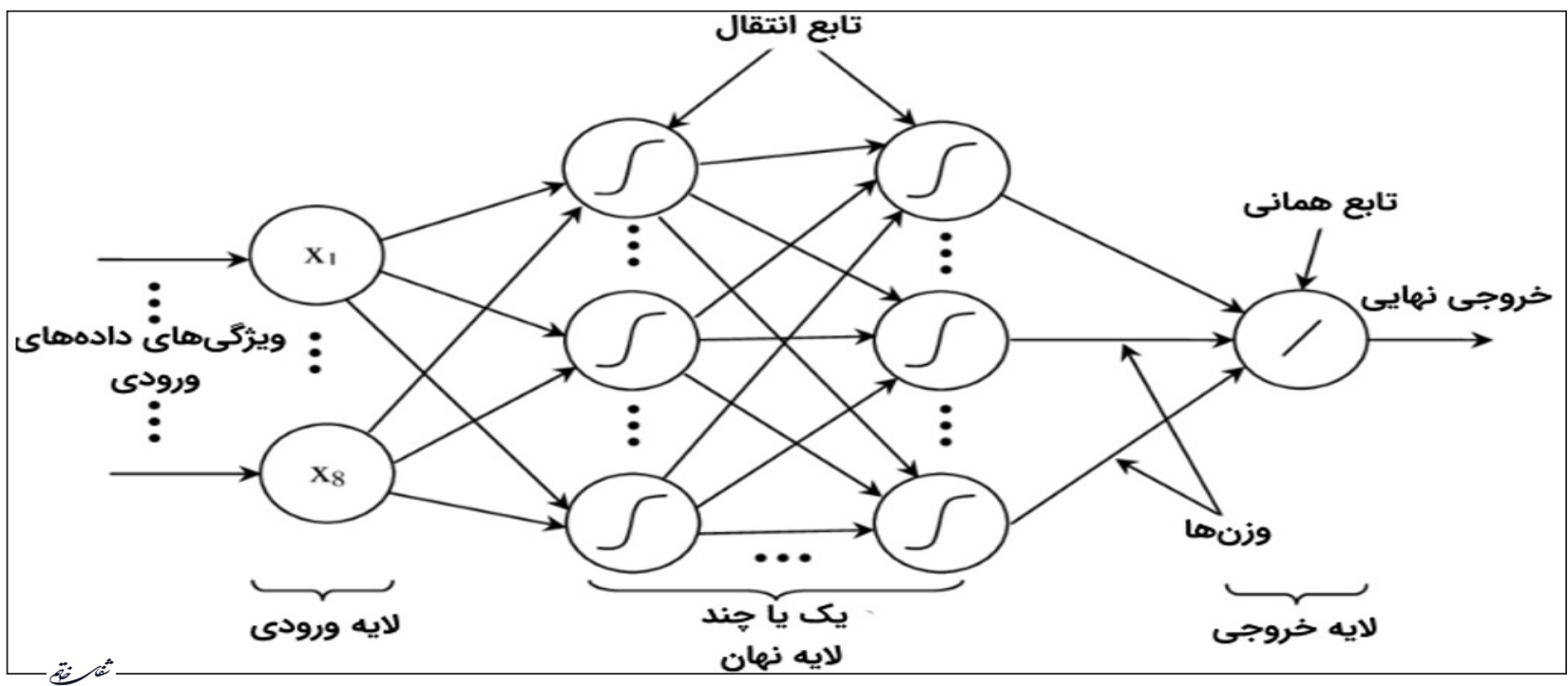

تصوير r- شبكة عصبى يرسيترون جند لايه 


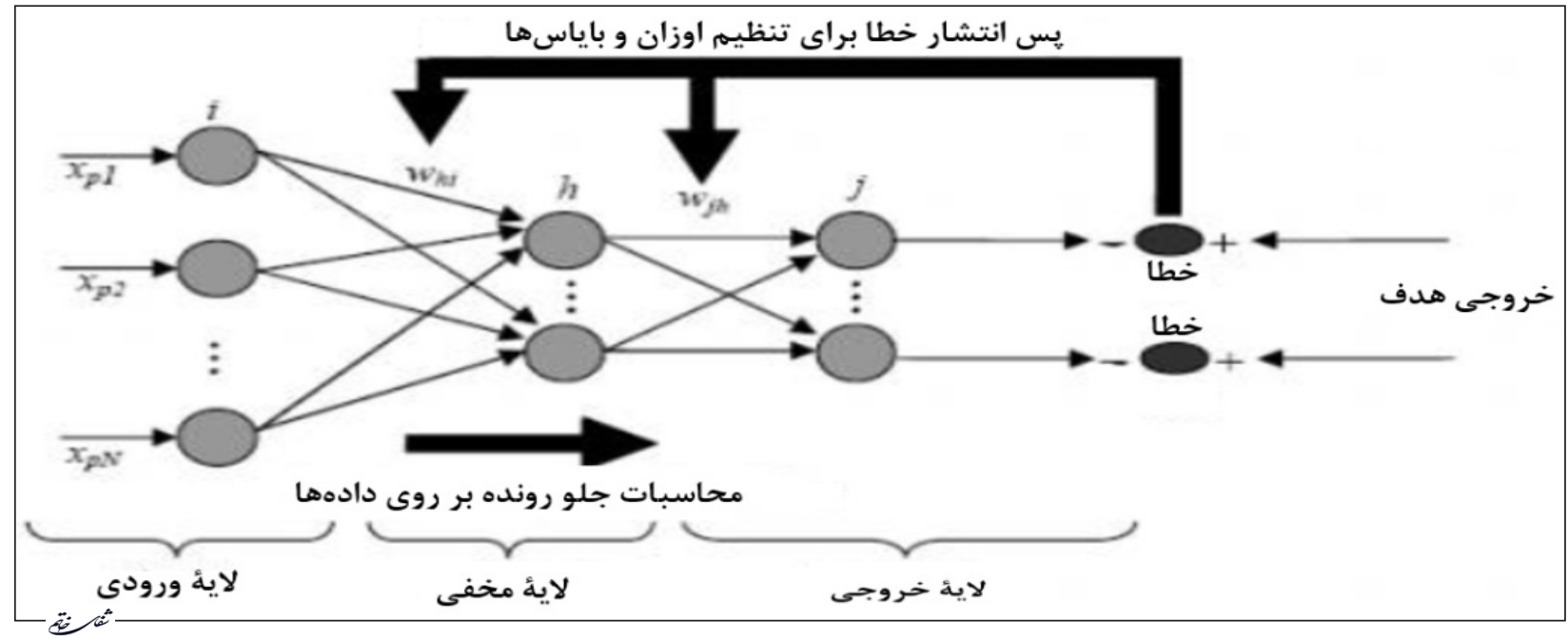

تصوير f- شبكة عصبى يس انتشار خطا

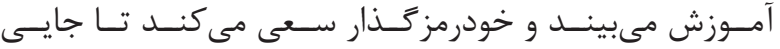

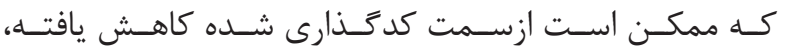

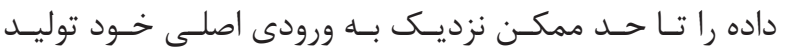

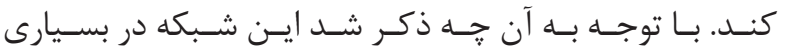

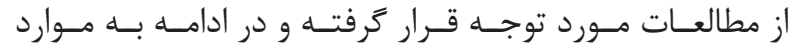

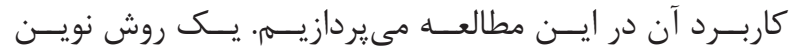

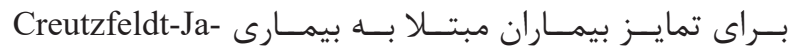

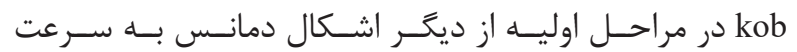

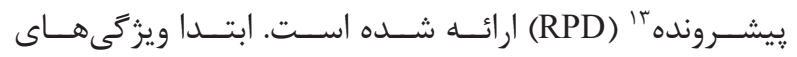

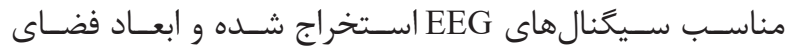

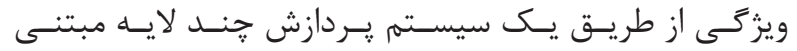

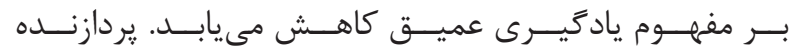

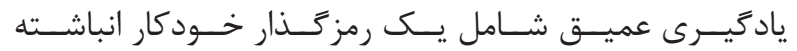

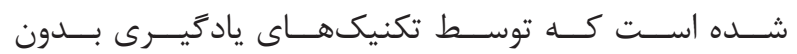

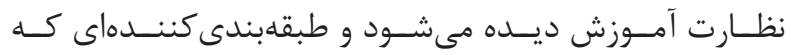

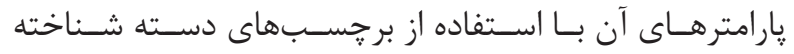

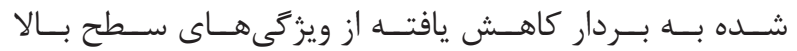

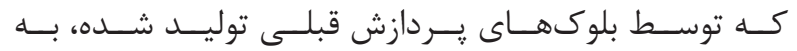

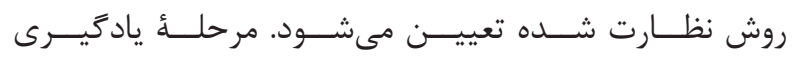

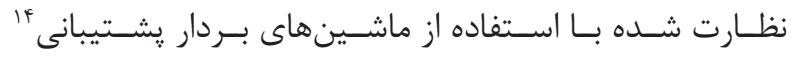

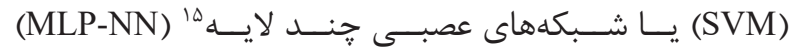

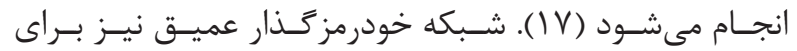

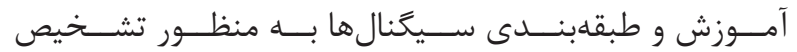

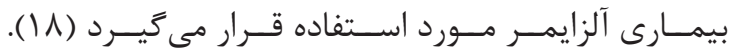

\section{شبكة عصبى كانولوشن}

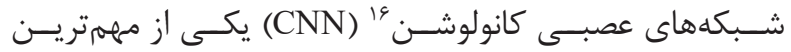

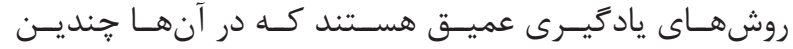

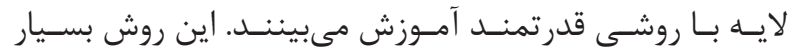

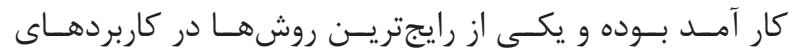

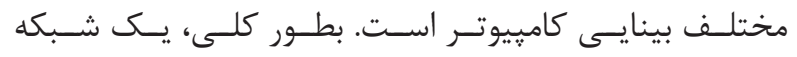

${ }^{7}$ Convolution neural network

${ }^{8}$ Autoencoder neural network

${ }^{9}$ Restricted Boltzmann Machines

${ }^{10}$ Sparse Coding

${ }^{1}$ Dimensionality reduction
شبكة عصبى عميق

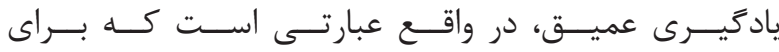

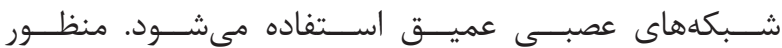

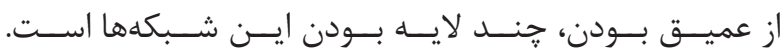

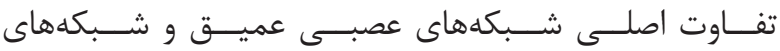

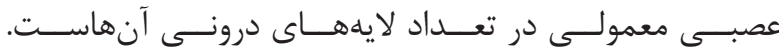

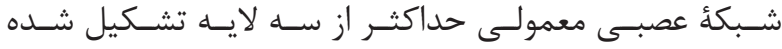

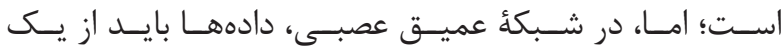

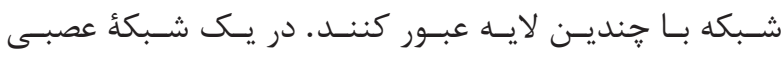

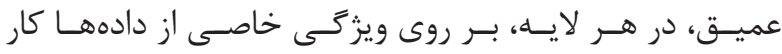

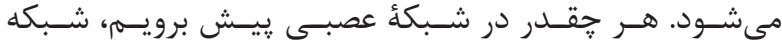

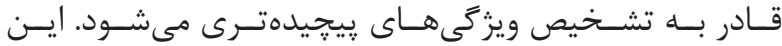

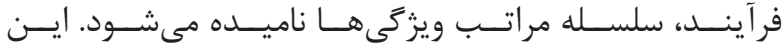

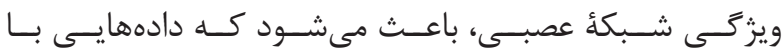

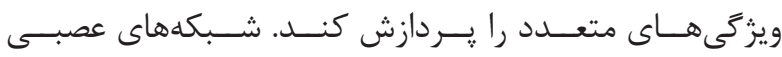

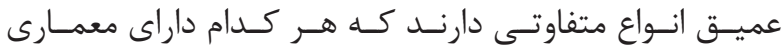

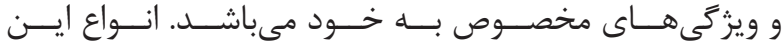

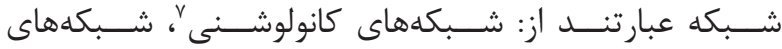

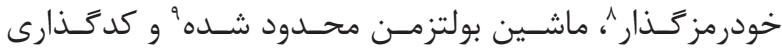

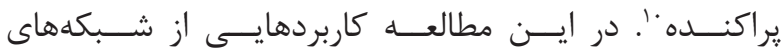

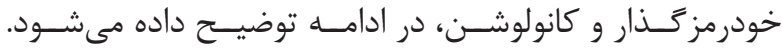

\section{شبكةُ عصبى خودرمزكذار}

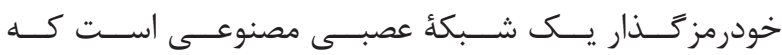

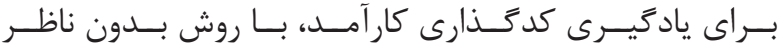

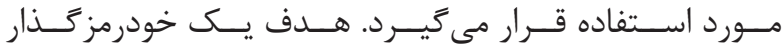

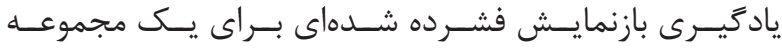

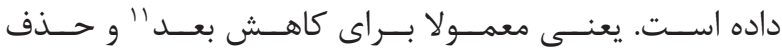

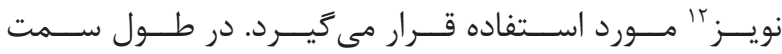

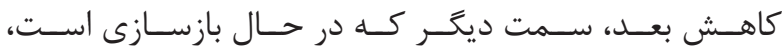

\footnotetext{
${ }^{12}$ Noise

${ }^{13}$ Rapidly progressive dementia; RPD

${ }^{14}$ Support vector machine; SVM

${ }^{15}$ Multilayer neural networks; MLP-NN

${ }^{16}$ Convolution neural network; $\mathrm{CNN}$
} 

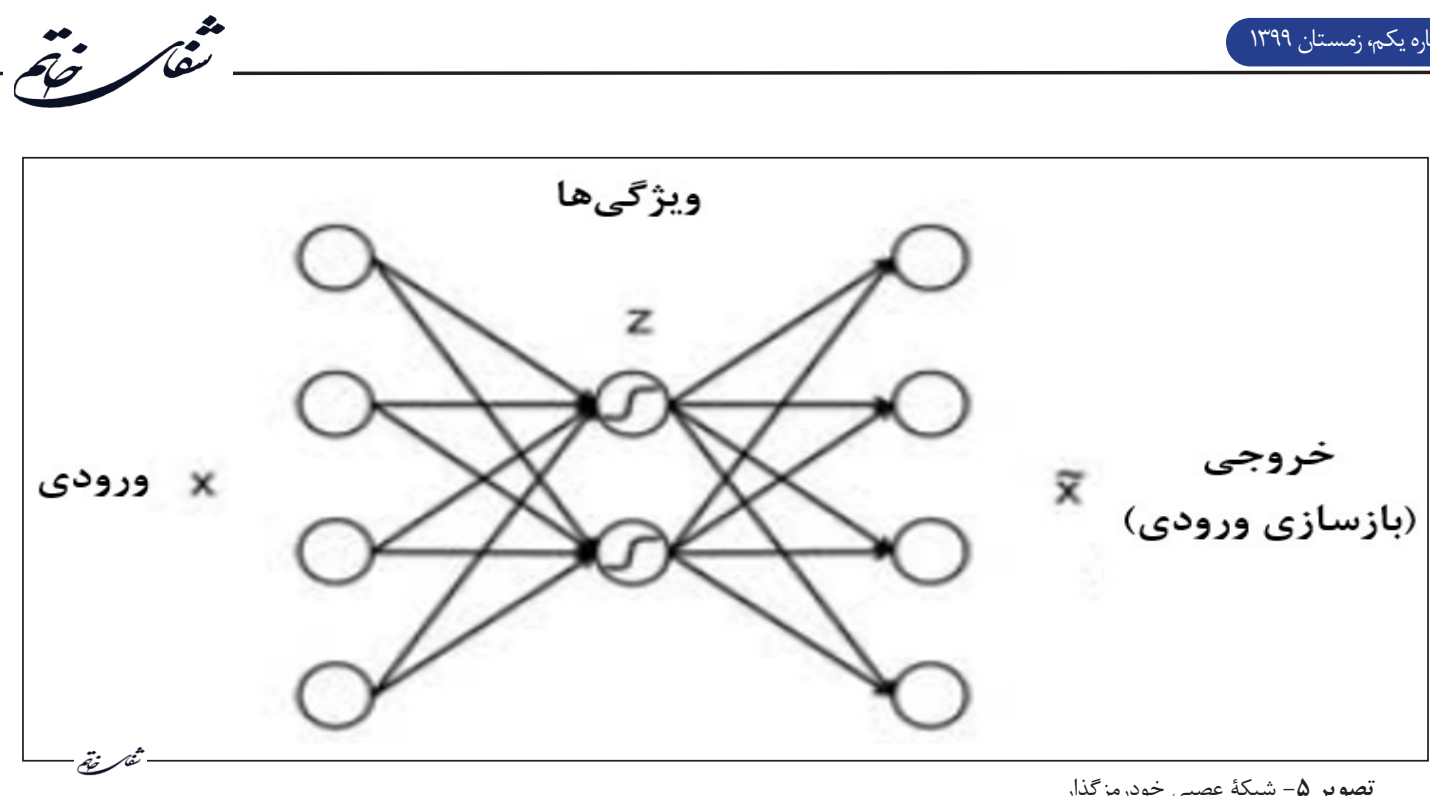

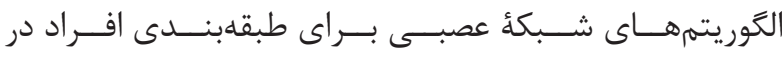

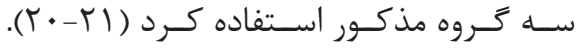

شبكة عصبى مصنوعى با الكوريته زنتيك(GA/ANN)

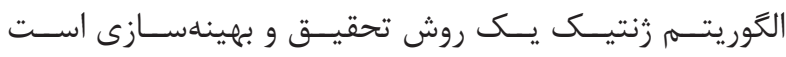

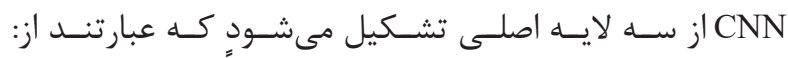

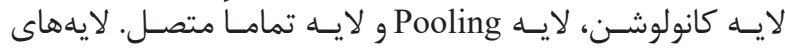

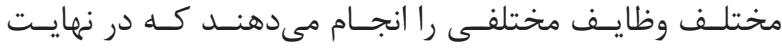

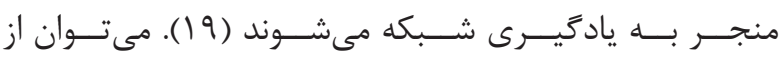

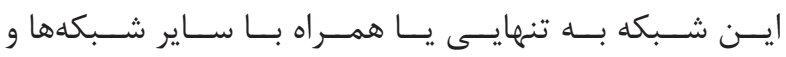

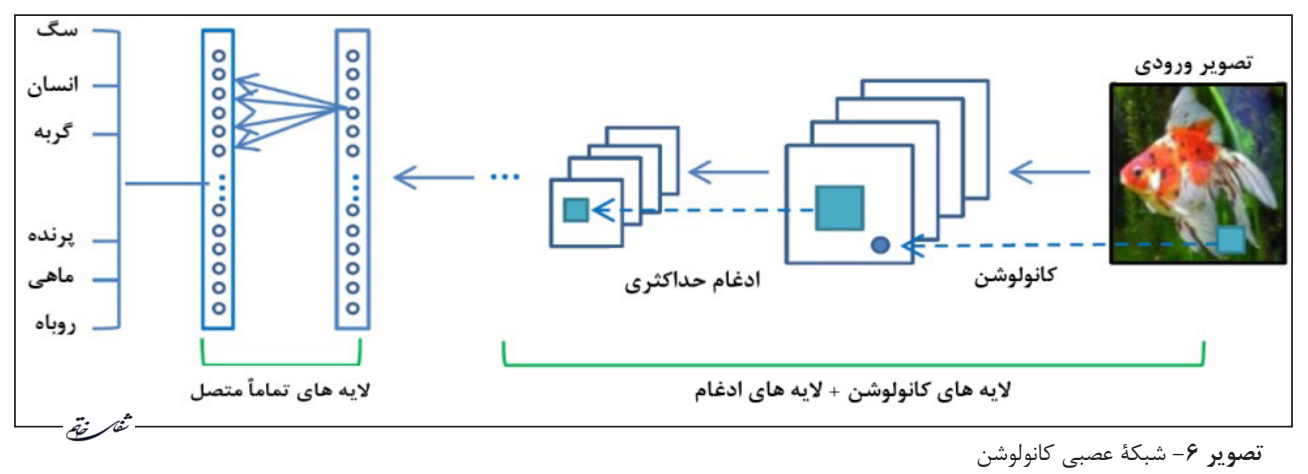

شبكة عصبى NeuCube

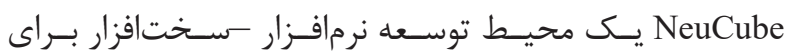

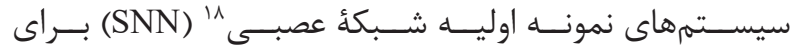

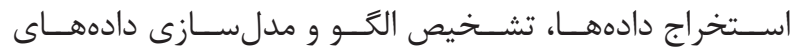

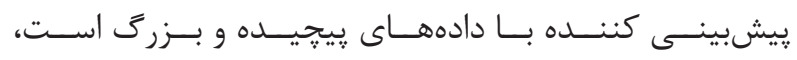

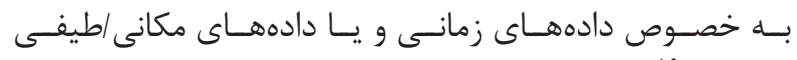

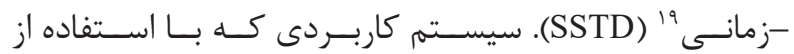

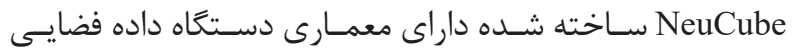

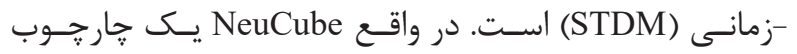

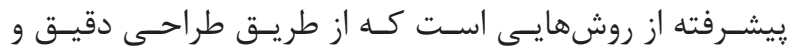

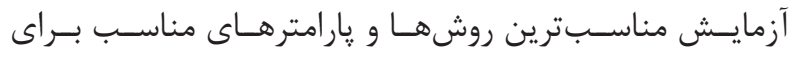

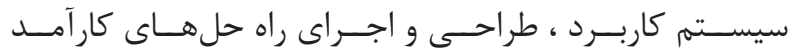

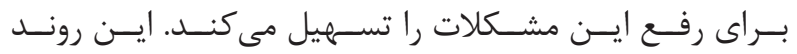

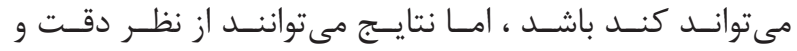

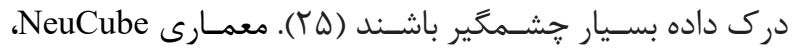

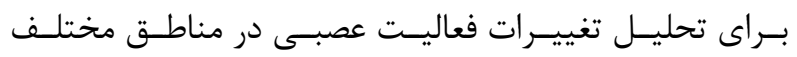

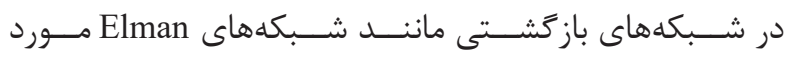

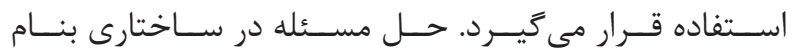

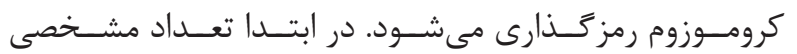

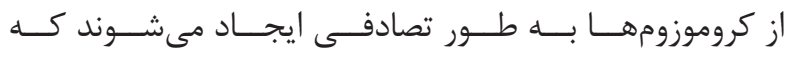

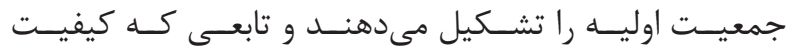

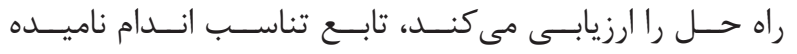

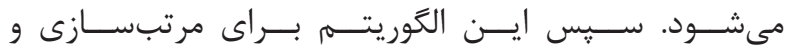

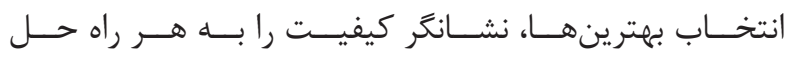

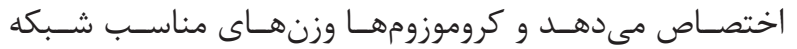

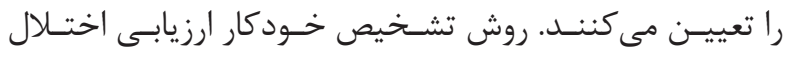

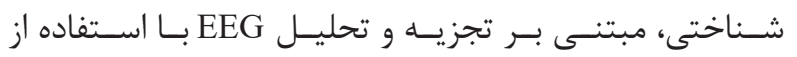

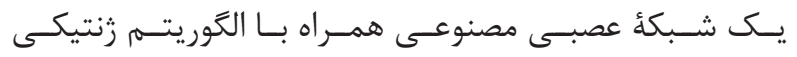

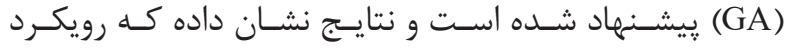

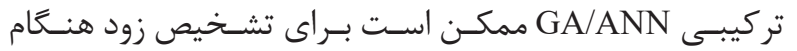

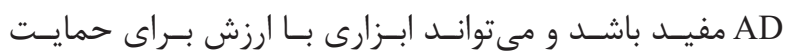

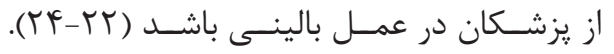




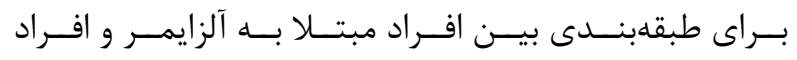

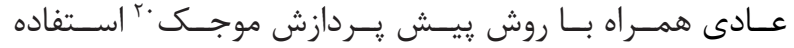

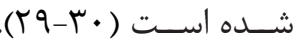
شبكة عصبى متناقض

شـبكةٔ عصبـى مصنوعَى متناقـض "r (PANN) يـك سـاختار

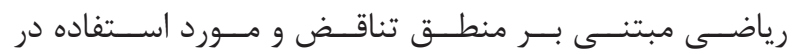

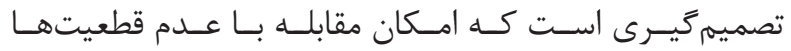

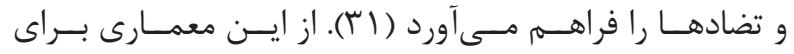

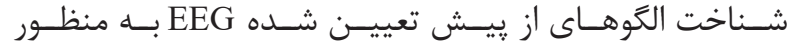

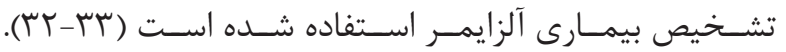

$$
\text { شبكة عصبى احتمالى }
$$

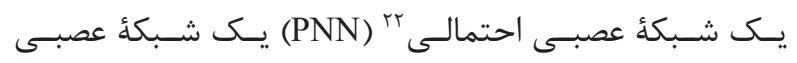

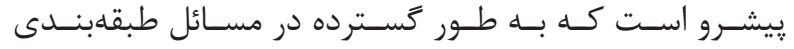

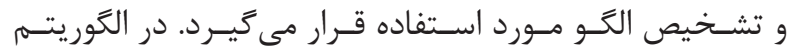

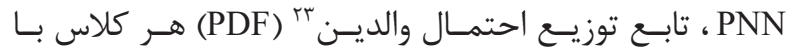

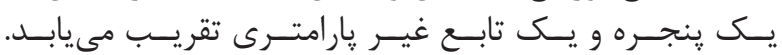

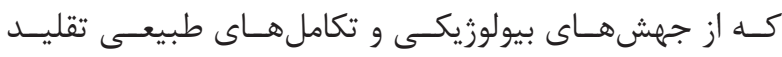

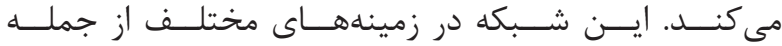

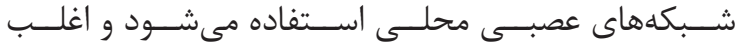

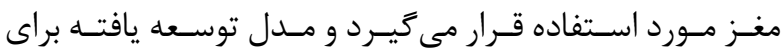

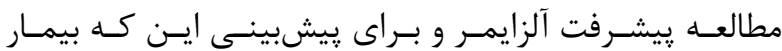

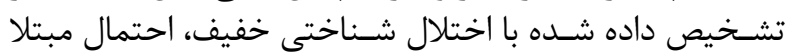

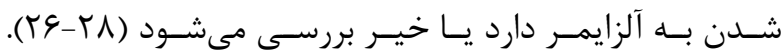
شبكة عصبى بازگشتى

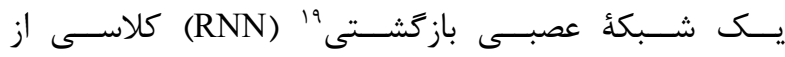

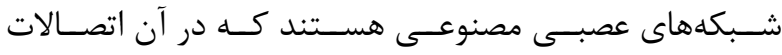

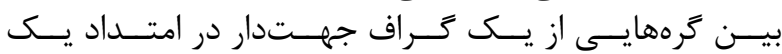

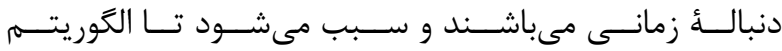

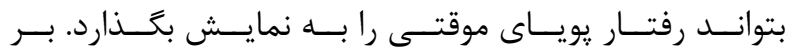

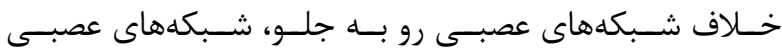

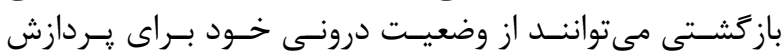

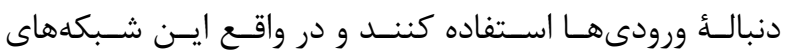

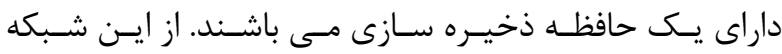

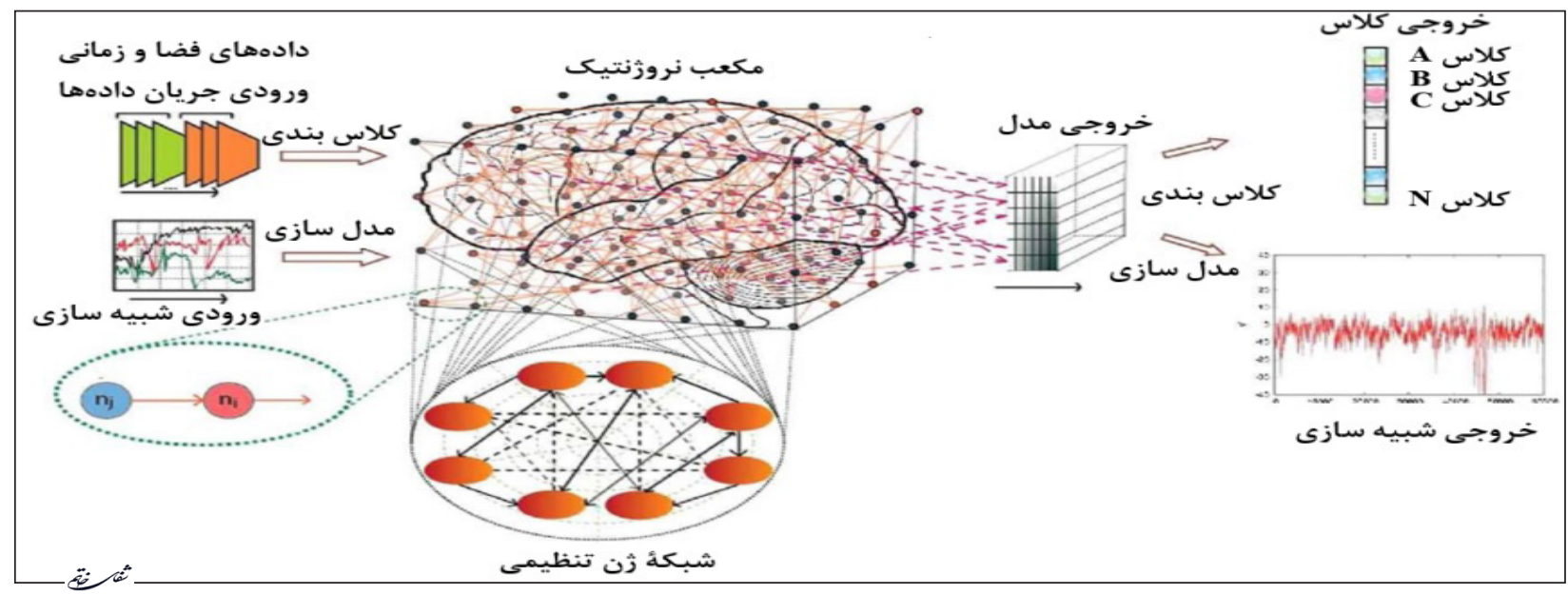

تصوير V- شبكة عصبى NeuCube

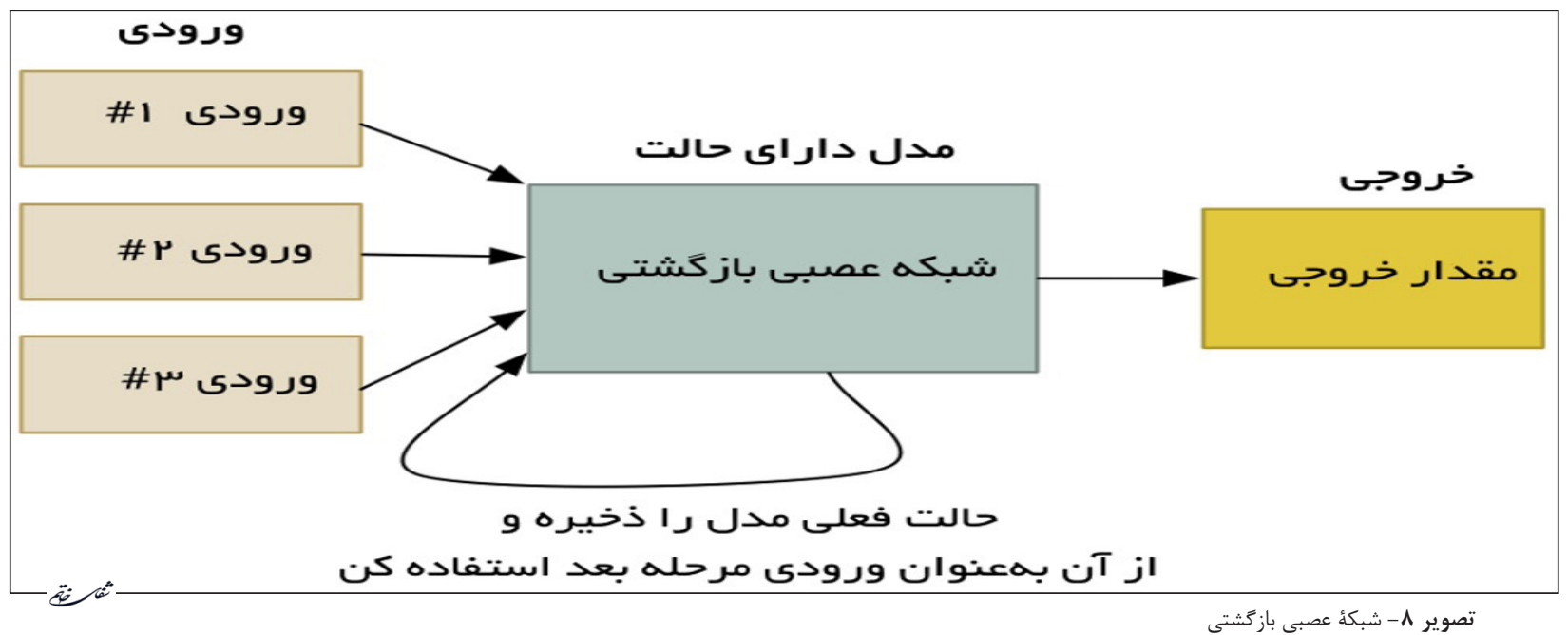

${ }^{19}$ Recurrent neural network; RNN

${ }^{20}$ Wavelet preprocessing

${ }^{21}$ Paraconsistent artificial neural network; PANN
${ }^{22}$ Probabilistic neural network; PNN

${ }^{23}$ Parent distribution function; PDF 


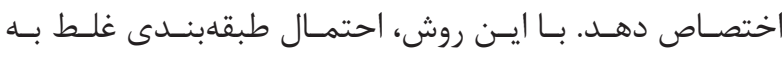

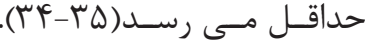

شبكة عصبى مuzzy

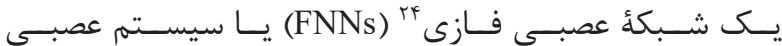

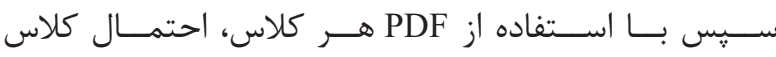

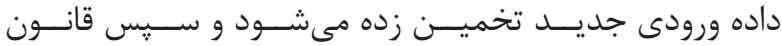

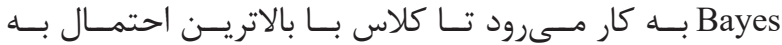

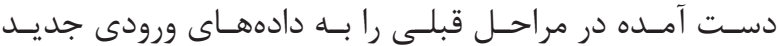

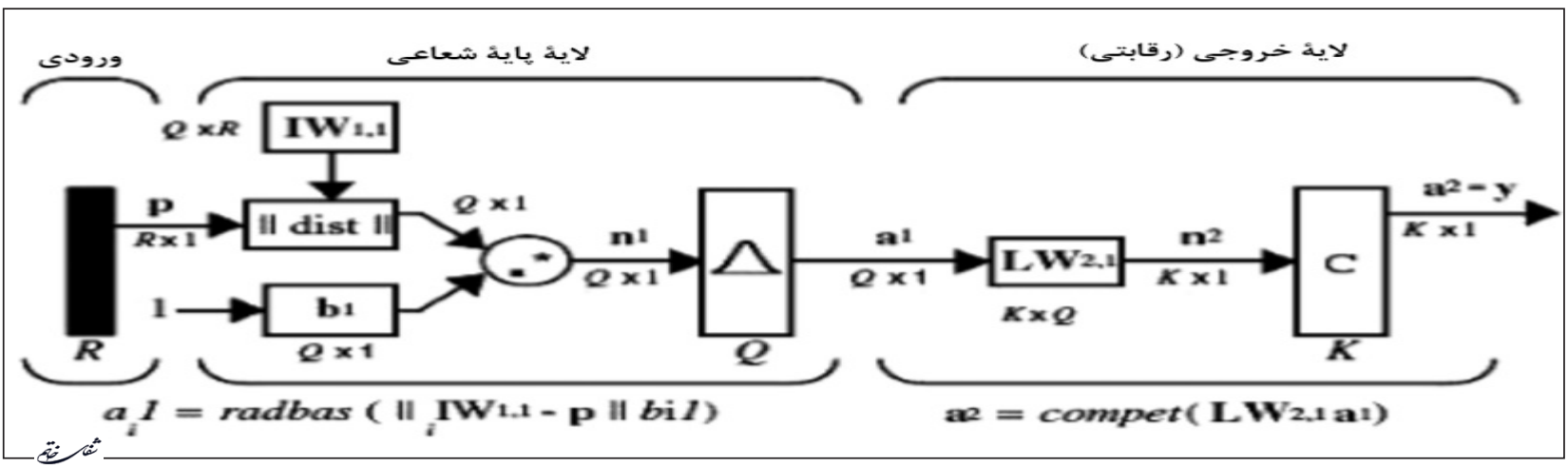

تصوير 9: شبكأ عصبى احتمالى

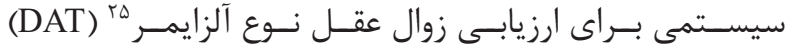

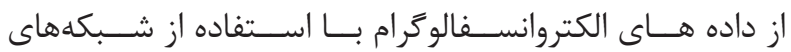

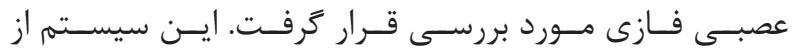

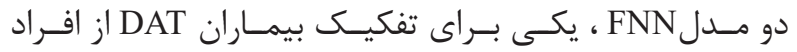

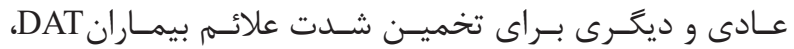

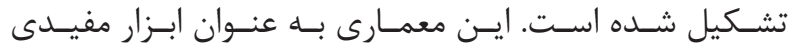

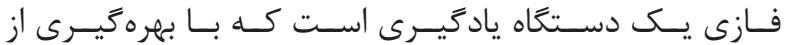

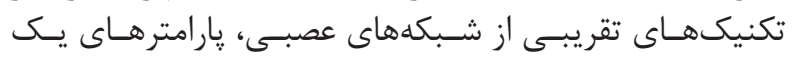

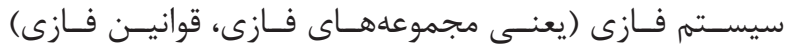

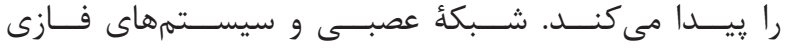

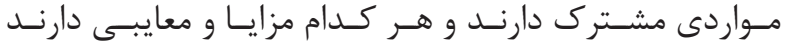

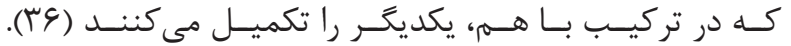

جدول ا- انواع شبكههاى عصبى مورد مطالعه

\begin{tabular}{|c|c|c|}
\hline 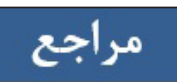 & 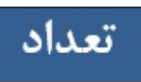 & شبكة عصبى مورد استفاده \\
\hline$(9-1 \cdot)$ & ه مقاله & يرسيترون تك لايه \\
\hline$(|1-| f)$ & 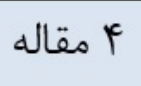 & يرسيترون جند لايه \\
\hline$(r-Q)$ & 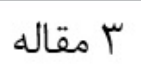 & شبكةُ عصبى ريشخور \\
\hline$(t r-t)^{2}$ & 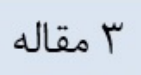 & شبكةُ عصبى مصنوعى با الكوريتم زنتيك (GA/ANN) \\
\hline$($ (YE-YN) & 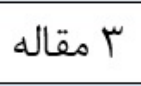 & شبكةُ عصبى NeuCube \\
\hline$(10-19)$ & 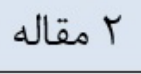 & شبكةُ يس انتشار خطا \\
\hline$(1 V-11)$ & 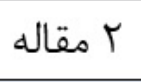 & شبكةُ عصبى خودرمزخذار (AE) \\
\hline$(r \cdot-41)$ & 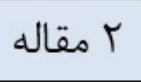 & شبكة عصبى كانولوشن (CNN) \\
\hline$($ (†-ケ•) & 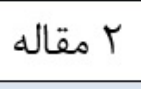 & شبكةُ عصبى بازَشتى (RNN) \\
\hline (זr-זr) & 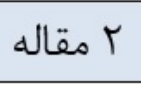 & شبكة عصبى متناقض (PAN) \\
\hline$(T \omega)$ & ا مقاله & شبكةُ عصبى احتمالى (PNN) \\
\hline$(T V)$ & ا مقاله & شبكةُ عصبى فازى (FNN) \\
\hline
\end{tabular}

${ }^{24}$ Fuzzy neural networks; FNNs

${ }^{25}$ Dementia of the Alzheimer type; DAT 


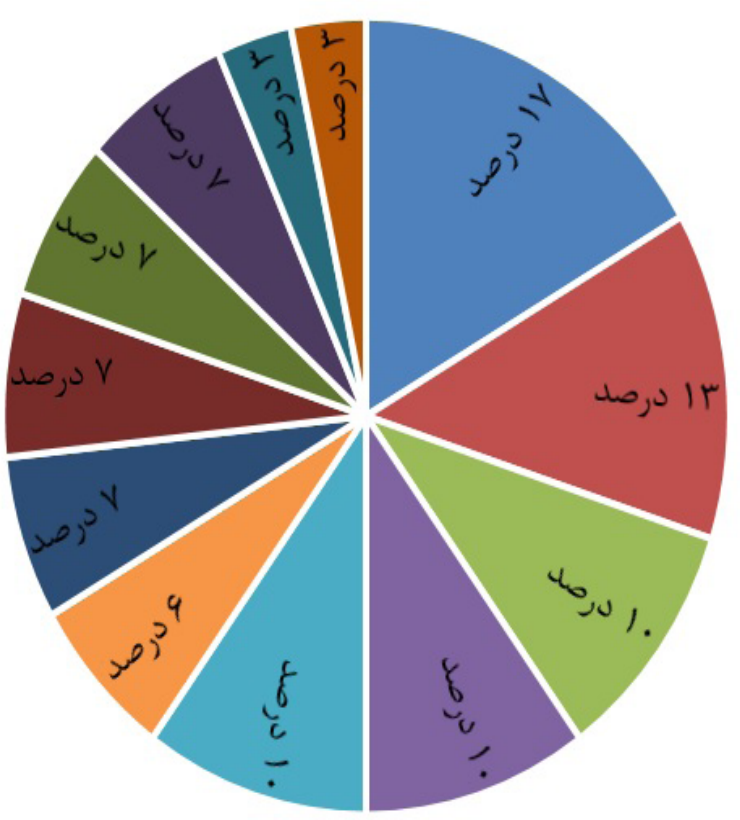

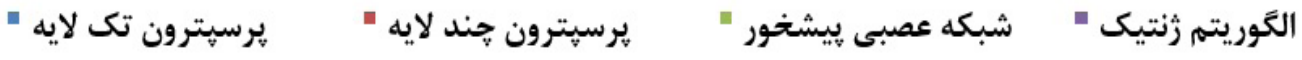

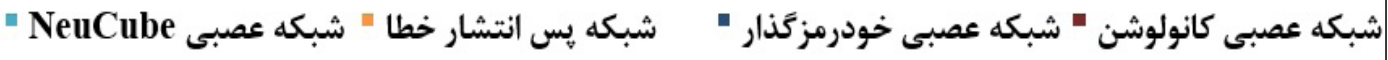

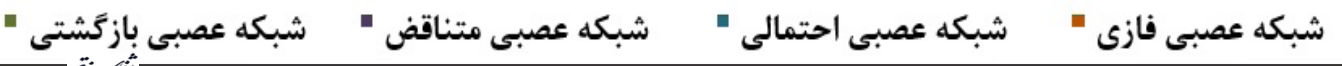
نمودار ا- مقايسه تعداد انواع شبكهاى عصبى مورد استفاده در مطالعات

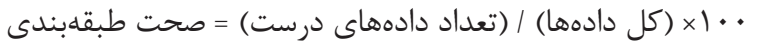

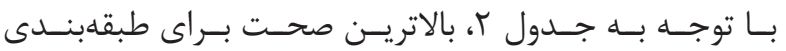

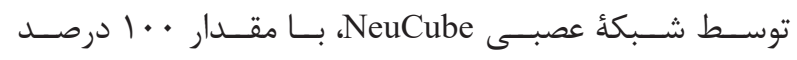

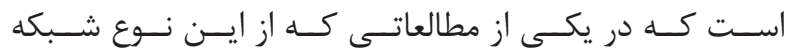

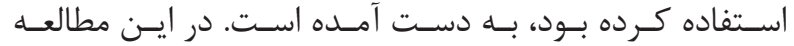

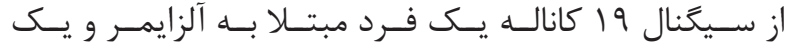

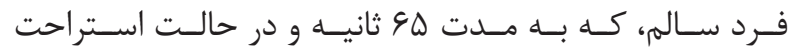

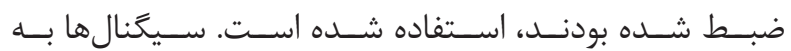

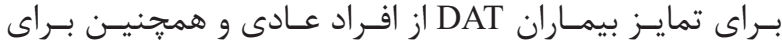

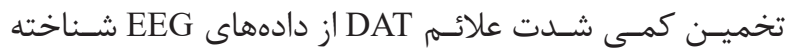

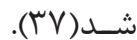

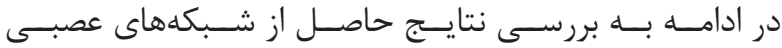

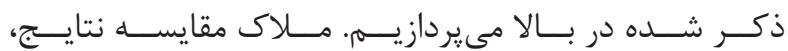

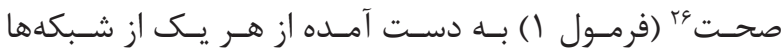

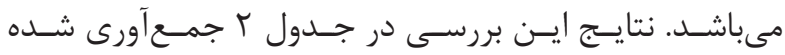
اسـت ابت

جدول r- دقت طبقهبندى انواع شبكههاى عصبى مصنوعى

\begin{tabular}{|c|c|c|}
\hline ساير روشىهاى مورد مقايسه در مطالعه & بازه دقت & شبكة عصبى مورر استنفاده \\
\hline ماشين بردار يشتيبان، ركرسيون منطقى، درخت تصميمه & ا A & يرسبترون تى لايه \\
\hline- & 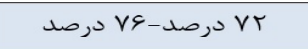 & يرسيترون جند لايه \\
\hline ركرسيون، ماشين بردار يشتيبان & १ هرصد-ه & شبكةُ عصبى بيشخور \\
\hline- & 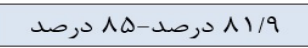 & شبكأ عصبى مصنوعى با الكوريتم زنتيك (GA/ANN) \\
\hline ماشين بردار هيشتيبان & | & شبكئ عصبى NeuCube \\
\hline- & 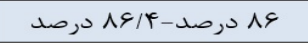 & شبكؤ يس انتشار خطا \\
\hline ماشين برقار يشتيبان، يرسيترون جند لايه & 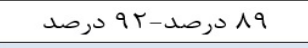 & شبكةُ عصبى خودرمزَخذار (AE) \\
\hline برسيترون بيشخور جند لايد & ا & شبكأ عصبى كانولوشن (CNN) \\
\hline يبش يردازش موجك & ل م درصد-19 درصد & شبكأ عصبى بازگشتى (RNN) \\
\hline- & ل م درصد-1 م مرصد & شبكةُ عصبى متناقض (PAN) \\
\hline- & 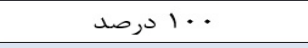 & شبكأ عصبى احتمالى (PNN) \\
\hline- & ( 9 . & شبكأ عصبى فازى (FNN) \\
\hline
\end{tabular}




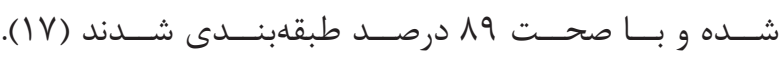

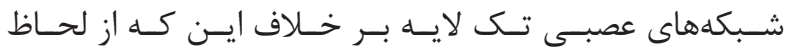

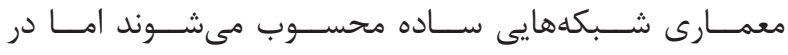

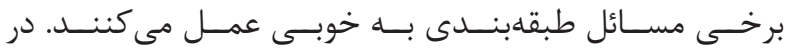

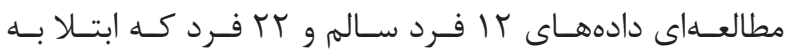

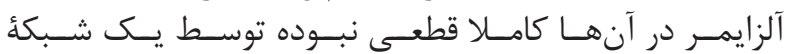

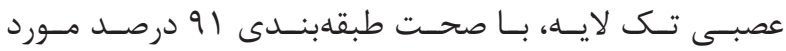

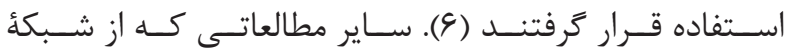

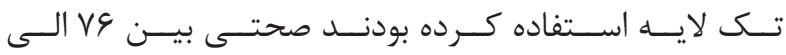

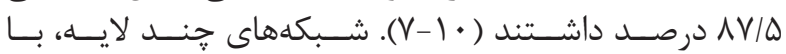

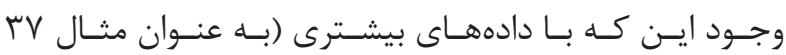

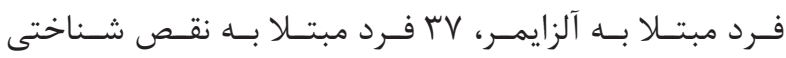

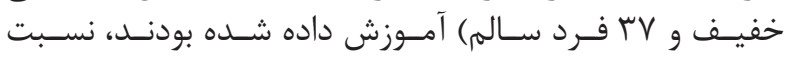

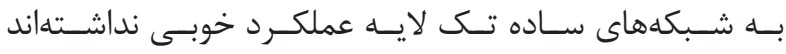

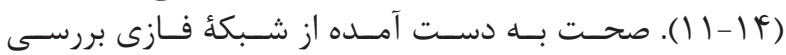

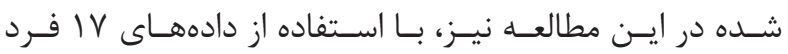

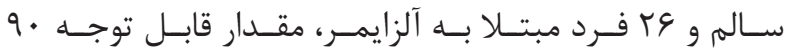

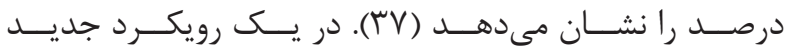

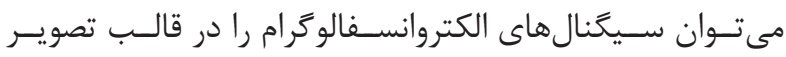

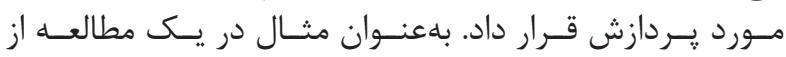

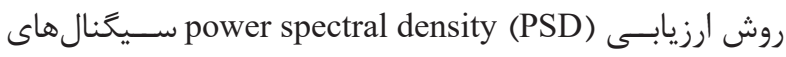

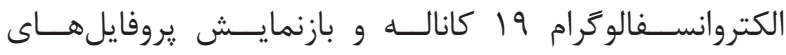

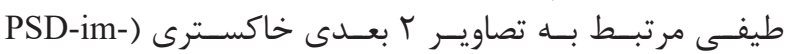

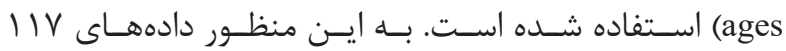

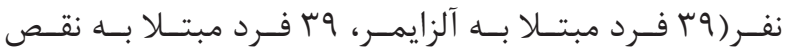

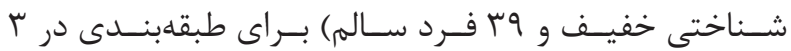

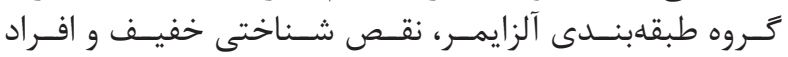

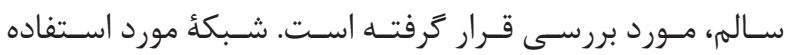

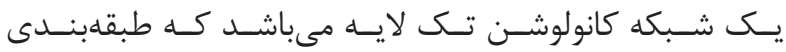

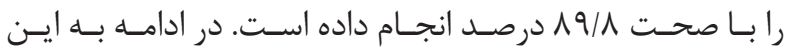

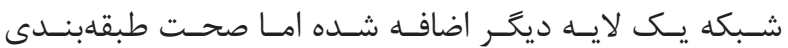

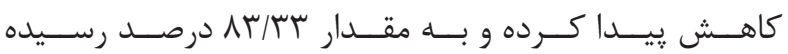

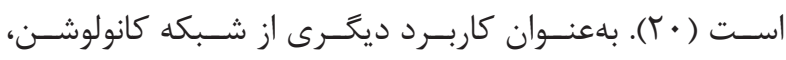

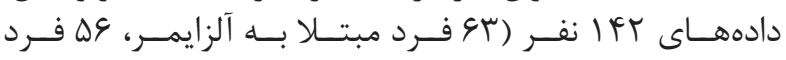

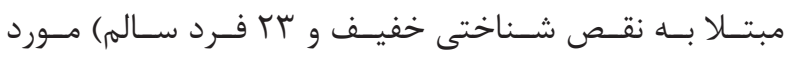

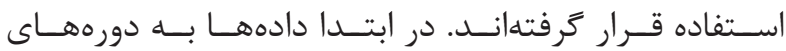
(epoch)

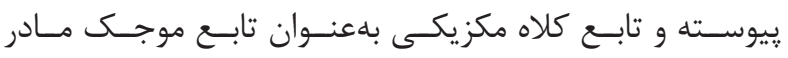

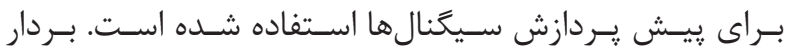

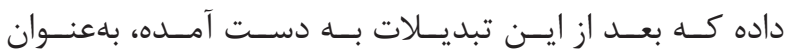

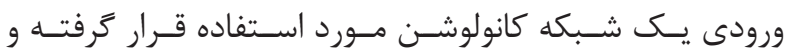

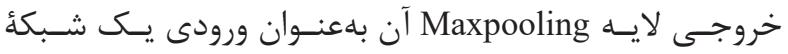

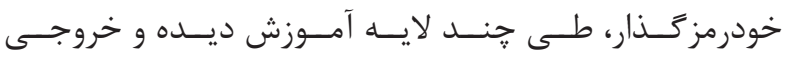

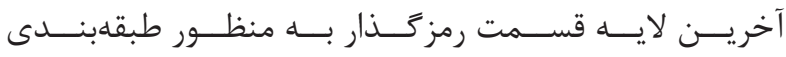

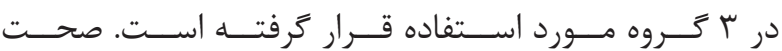

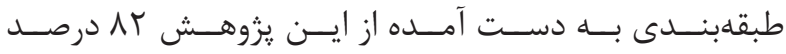

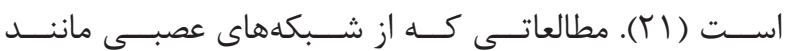

${ }^{27}$ Continuous wavelet transform; CWT

${ }^{28}$ Sensitivity

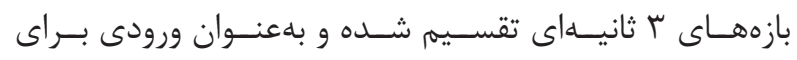

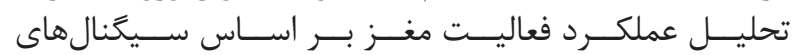

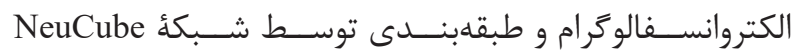

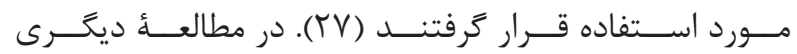

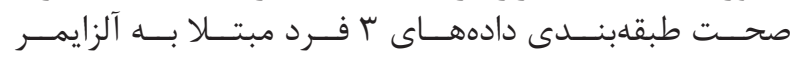

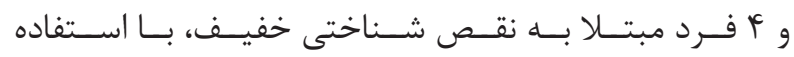

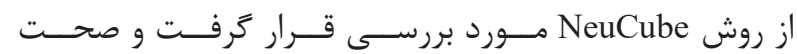

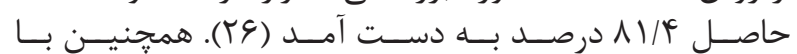

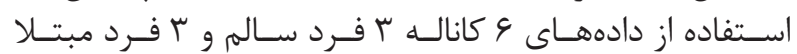

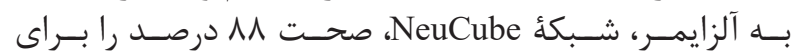

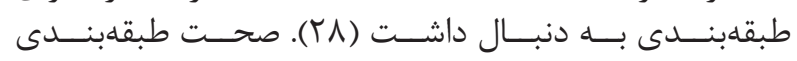

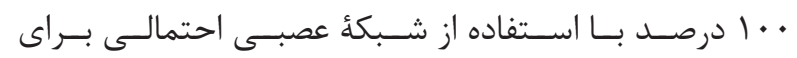

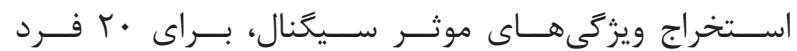

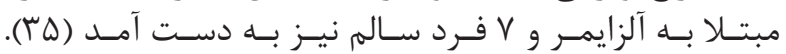

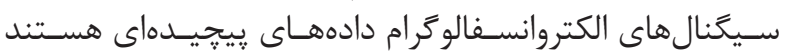

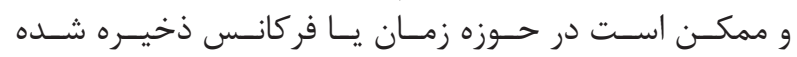

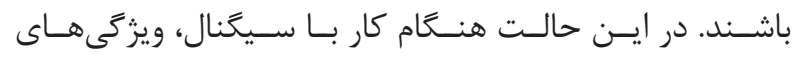

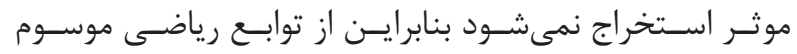

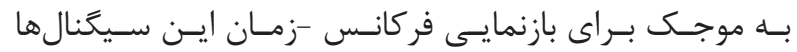

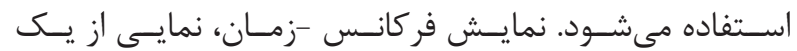

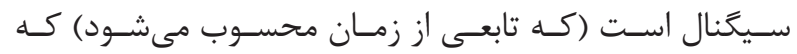

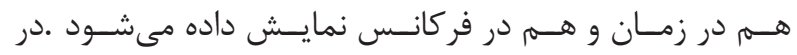

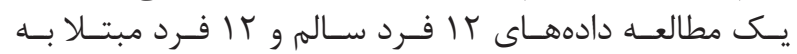

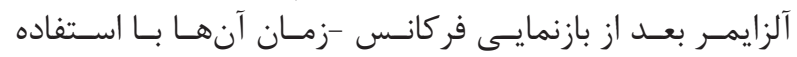

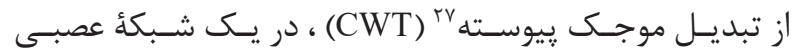

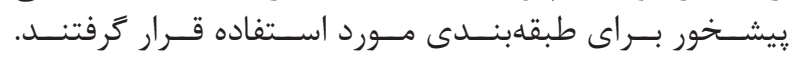

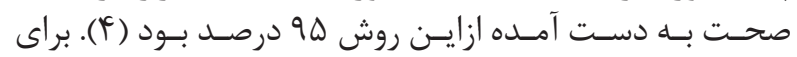

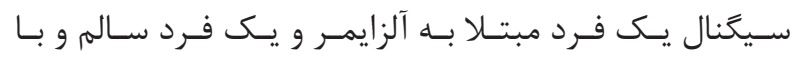

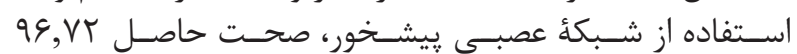

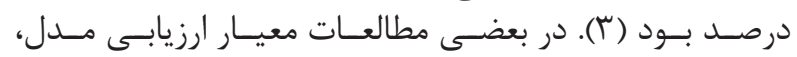

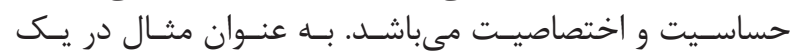

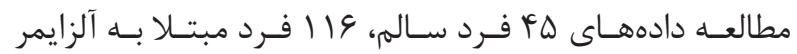

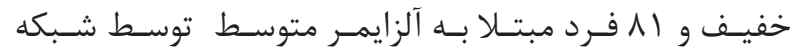

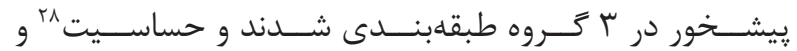

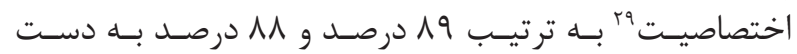

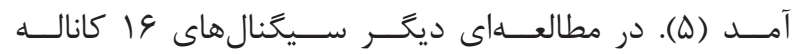

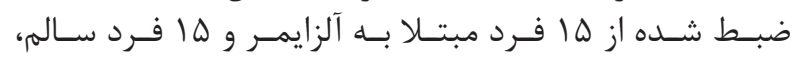

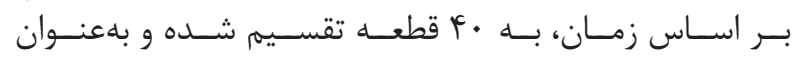

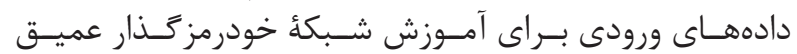

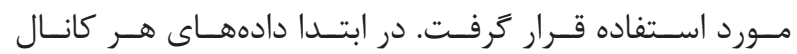

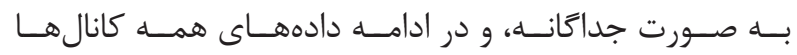

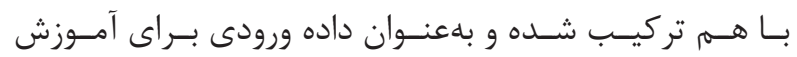

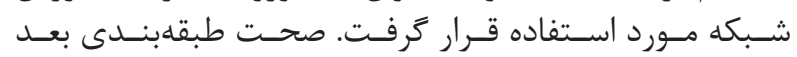

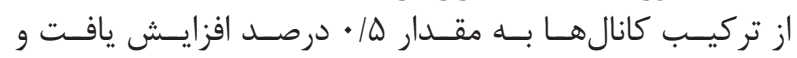

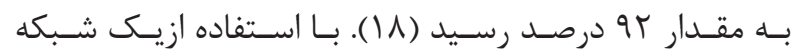

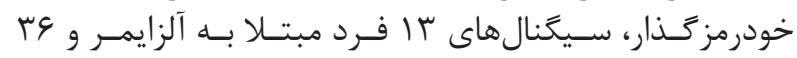

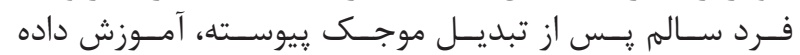

${ }^{29}$ Specificity 


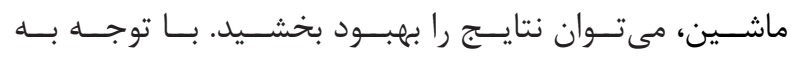

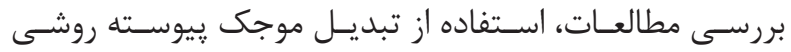

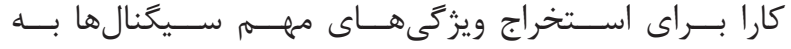

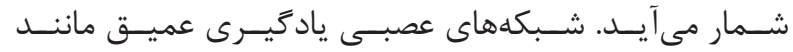

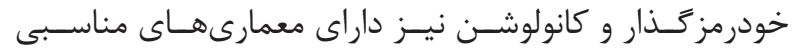

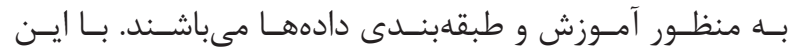

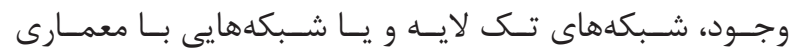

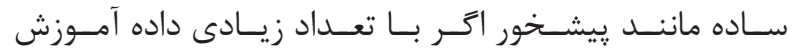

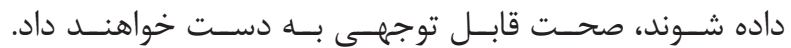

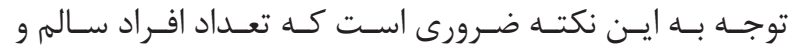

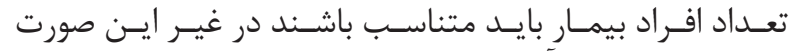

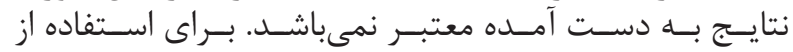

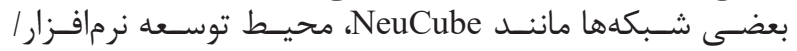

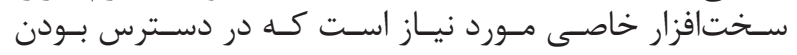

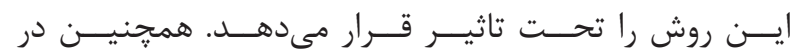

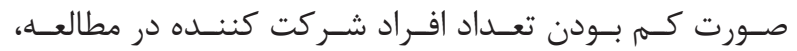

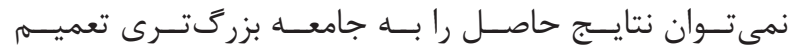

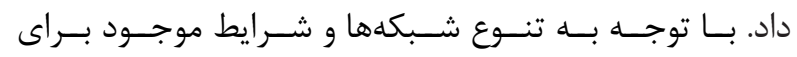

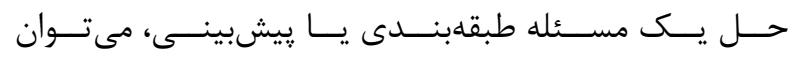

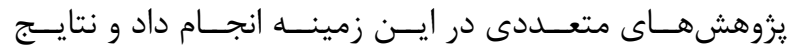

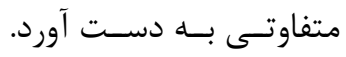

1. Mayeux R, Stern Y. Epidemiology of Alzheimer disease. Cold Spring Harbor Perspect. Med. 2012; 2(8): a006239.

2. Agatonovic-Kustrin S, Beresford R. Basic concepts of artificial neural network (ANN) modeling and its application in pharmaceutical research. J. Pharm. Biomed. Anal. 2000; 22(5): 717-27.

3. Schetinin V. Polynomial Neural Networks Learnt to Classify EEG Signals. 2001.

4. Puig D, Jayapathy R, Mohandhas B, Lazar P, Rathnam MR, Torrents-Barrena J. Complex wavelet algorithm for computer-aided diagnosis of Alzheimer's disease. Electron. Lett. 2015; 51.

5. Lehmann C, Koenig T, Jelic V, Prichep L, John RE, Wahlund LO, et al. Application and comparison of classification algorithms for recognition of Alzheimer's disease in electrical brain activity (EEG). J. Neurosci. Methods. 2007; 161(2): 342-50.

6. Trambaiolli LR, Falk TH, Fraga FJ, Anghinah R, Lorena AC. EEG spectro-temporal modulation energy: a new feature for automated diagnosis of Alzheimer's disease. Conf Proc IEEE Eng Med Biol Soc. 2011;2011:3828-31.

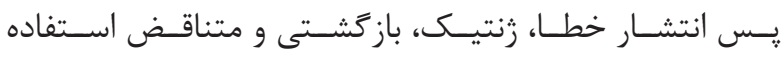

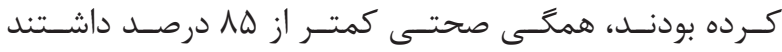

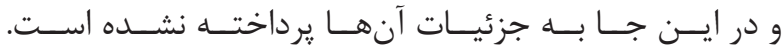
بحث و نتيجه كيرى

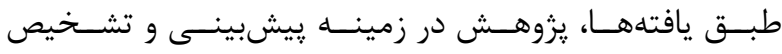

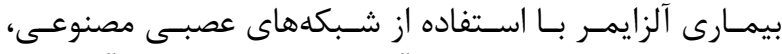

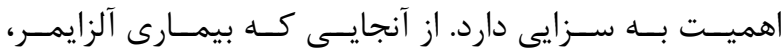

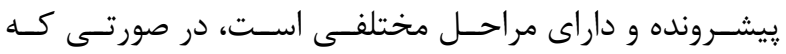

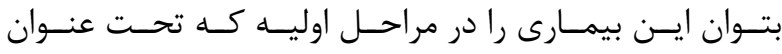

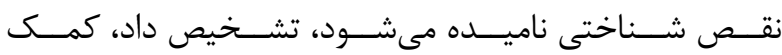

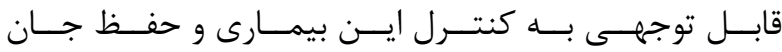

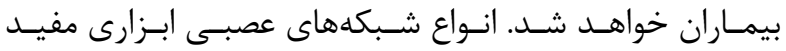

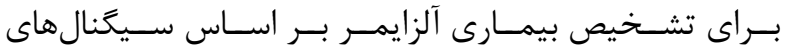

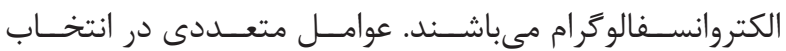

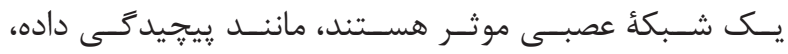

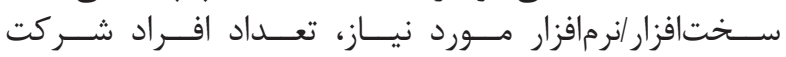

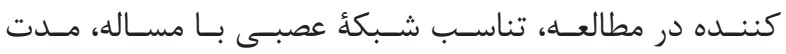

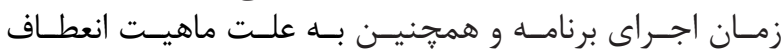

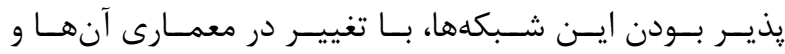

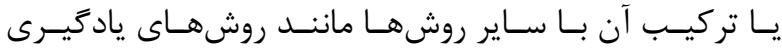
منابع

7. McBride J, Zhao X, Munro N, Jiang Y, Smith C, Jicha $\mathrm{G}$, editors. Scalp EEG signal reconstruction for detection of mild cognitive impairment and early Alzheimer's disease. 2013 Biomedical Sciences and Engineering Conference (BSEC); 2013 MAy 21-23.

8. Rodrigues P, Teixeira J, Homero R, Poza J, Carreres A. Classification of Alzheimer^s Electroencephalograms using Artificial Neural Networks and Logistic Regression 2011.

9. Hudson DL, Cohen ME, Kramer M, Szeri A, Chang FL, editors. Diagnostic Implications of EEG Analysis in Patients with Dementia. Conference Proceedings 2nd International IEEE EMBS Conference on Neural Engineering, 2005; 16-19.

10. Triggiani AI, Bevilacqua V, Brunetti A, Lizio R, Tattoli G, Cassano F, et al. Classification of Healthy Subjects and Alzheimer's Disease Patients with Dementia from Cortical Sources of Resting State EEG Rhythms: A Study Using Artificial Neural Networks. Front. Neurosci. 2016; 10:604.

11. Kim D, Kim K. Detection of Early Stage Alzheimer's Disease using EEG Relative Power with Deep Neural Network. Conf Proc IEEE Eng Med Biol Soc. 2018; 2018: 352-5.

12. Ventouras EM, Economou NT, Kritikou I, Tsekou H, 


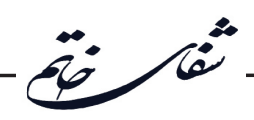

Paparrigopoulos TJ, Ktonas PY. Performance evaluation of an Artificial Neural Network automatic spindle detection system. Conf Proc IEEE Eng Med Biol Soc. 2012; 2012: 4328-31.

13. Ruiz-Gomez S, Gómez C, Poza J, GutiérrezTobal G, Tola-Arribas M, Cano M, et al. Automated Multiclass Classification of Spontaneous EEG Activity in Alzheimer's Disease and Mild Cognitive Impairment. Entropy. 2018; 20.

14. Rodrigues P, Teixeira J. Alzheimer's disease recognition with artificial neural networks. 2013. p. 102-18.

15. Bevilacqua V, Salatino AA, Leo CD, Tattoli G, Buongiorno D, Signorile D, et al., editors. Advanced classification of Alzheimer's disease and healthy subjects based on EEG markers. 2015 International Joint Conference on Neural Networks (IJCNN); 2015 12-17 July 2015.

16. Besthorn C, Zerfass R, Geiger-Kabisch C, Sattel H, Daniel S, Schreiter-Gasser U, et al. Discrimination of Alzheimer's disease and normal aging by EEG data. Electroencephalogr. Clin. Neurophysiol. 1997; 103(2): 241-8.

17. Morabito FC, Campolo M, Mammone N, Versaci M, Franceschetti S, Tagliavini F, et al. Deep Learning Representation from Electroencephalography of EarlyStage Creutzfeldt-Jakob Disease and Features for Differentiation from Rapidly Progressive Dementia. Int J Neural Syst. 2017; 27(2): 1650039.

18. Zhao Y, He L. Deep Learning in the EEG Diagnosis of Alzheimer's Disease2014. 340-53 p.

19. Zeiler MD, editor Hierarchical Convolutional Deep Learning in Computer Vision 2013.

20. Ieracitano C, Mammone N, Bramanti A, Hussain A, Morabito FC. A Convolutional Neural Network approach for classification of dementia stages based on 2D-spectral representation of EEG recordings. Neurocomputing. 2019; 323: 96-107.

21. Morabito FC, Campolo M, Ieracitano C, Ebadi JM, Bonanno L, Bramanti A, et al., editors. Deep convolutional neural networks for classification of mild cognitive impaired and Alzheimer's disease patients from scalp EEG recordings. 2016 IEEE 2nd International Forum on Research and Technologies for Society and
Industry Leveraging a better tomorrow (RTSI); 2016 Sept 7-9.

22. Berte F, Lamponi G, Calabro RS, Bramanti P. Elman neural network for the early identification of cognitive impairment in Alzheimer's disease. Funct Neurol. 2014; 29(1): 57-65.

23. Kim HT, Kim BY, Park EH, Kim JW, Hwang EW, Han SK, et al. Computerized recognition of Alzheimer disease-EEG using genetic algorithms and neural network. Future Generation Computer Systems. 2005; 21(7): 1124-30.

24. Cho S, Kim BY, Park EH, Chang YS, Kim J, Chung $\mathrm{K}$, et al. Automatic Recognition of Alzheimer's Disease Using Genetic Algorithms and Neural Network 2003. 695-702 p.

25. NeuCube Neurocomputing Software/Hardware Development Environment for Spiking Neural Network Applications in Data Mining, Pattern Recognition,and Predictive Data Modelling. Auckland, New Zealand: Knowledge Engineering and Discovery Research Institute 2016.

26. Capecci E, Doborjeh ZG, Mammone N, Foresta FL, Morabito FC, Kasabov N, editors. Longitudinal study of alzheimer's disease degeneration through EEG data analysis with a NeuCube spiking neural network model. 2016 International Joint Conference on Neural Networks (IJCNN); 2016 July 24-29.

27. Capecci E, Morabito F, Campolo M, Mammone N, Labate D, Kasabov N. A feasibility study of using the NeuCube spiking neural network architecture for modelling Alzheimer's disease and mild cognitive impairment EEG data2014.

28. Kasabov N, Capecci E. Spiking neural network methodology for modelling, classification and understanding of EEG spatio-temporal data measuring cognitive processes. Inf. Sci. (N. Y.). 2015; 294: 565-75.

29. Petrosian AA, Prokhorov DV, Lajara-Nanson W, Schiffer RB. Recurrent neural network-based approach for early recognition of Alzheimer's disease in EEG. Clin. Neurophysiol. 2001; 112(8): 1378-87.

30. Petrosian A, Prokhorov D, Schiffer R, editors. Recurrent neural network and wavelet transform based distinction between Alzheimer and control EEG. 
Proceedings of the First Joint BMES/EMBS Conference 1999 IEEE Engineering in Medicine and Biology 21st Annual Conference and the 1999 Annual Fall Meeting of the Biomedical Engineering Society (Cat N; 1999 1316 Oct. 1999.

31. Abe JM, Ortega NRS, Mário MC, Del Santo M, editors. Paraconsistent Artificial Neural Network: An Application in Cephalometric Analysis. KnowledgeBased Intelligent Information and Engineering Systems; 2005 2005//; Berlin, Heidelberg: Springer Berlin Heidelberg.

32. da Silva Lopes HF, Abe JM, Anghinah R. Application of paraconsistent artificial neural networks as a method of aid in the diagnosis of Alzheimer disease. NM/MIRD Pam. 2010; 34(6): 1073-81.

33. Abe JM, Lopes HFdS, Anghinah R. Paraconsistent artificial neural networks and Alzheimer disease: a preliminary study. Dement Neuropsychol. 2007; 1(3): 241-7.

34. Zeinali Y, Story B. Competitive probabilistic neural network. Integrated Computer-Aided Engineering. 2017; $24: 1-14$

35. Sankari Z, Adeli H. Probabilistic neural networks for diagnosis of Alzheimer's disease using conventional and wavelet coherence. J. Neurosci. Methods. 2011; 197(1): 165-70.

36. Kruse DR. Fuzzy neural network. Germany: Institute for Information and Communication Systems; 2008.

37. Hibino S, Hanai T, Nagata E, Matsubara M, Fukagawa K, Shirataki T, et al. Fuzzy Neural Network Model for Assessment of Alzheimer-Type Dementia. J. Chem. Eng. Jpn . 2001; 34: 936-42. 